\title{
The Conjugate Gradient Viscosity Approximation Algorithm for Split Generalized Equilibrium and Variational Inequality Problems
}

\author{
Meixia Li $(\mathbb{D}$, Haitao Che $(\mathbb{D}$, and Jingjing Tan \\ School of Mathematics and Information Science, Weifang University, Weifang, Shandong 261061, China \\ Correspondence should be addressed to Meixia Li; limeixia001@163.com
}

Received 16 July 2018; Revised 28 September 2018; Accepted 15 October 2018; Published 1 November 2018

Academic Editor: Liguang Wang

Copyright (c) 2018 Meixia Li et al. This is an open access article distributed under the Creative Commons Attribution License, which permits unrestricted use, distribution, and reproduction in any medium, provided the original work is properly cited.

In this paper, we study a kind of conjugate gradient viscosity approximation algorithm for finding a common solution of split generalized equilibrium problem and variational inequality problem. Under mild conditions, we prove that the sequence generated by the proposed iterative algorithm converges strongly to the common solution. The conclusion presented in this paper is the generalization, extension, and supplement of the previously known results in the corresponding references. Some numerical results are illustrated to show the feasibility and efficiency of the proposed algorithm.

\section{Introduction}

Let $H_{1}$ and $H_{2}$ be real Hilbert spaces with inner product $\langle\cdot, \cdot\rangle$ and norm $\|\cdot\|$. Let $C$ and $Q$ be nonempty closed convex subsets of $H_{1}$ and $H_{2}$, respectively. Let $\left\{x_{n}\right\}$ be a sequence in $H_{1}$; then $x_{n} \longrightarrow x$ (respectively, $x_{n} \rightarrow x$ ) will denote strong (respectively, weak) convergence of the sequence $\left\{x_{n}\right\}$. Assume $w_{\omega}\left(x_{k}\right)=\left\{x: \exists x_{k_{j}} \rightarrow x\right\}$ to stand for the weak $\omega$ limit set of $x_{k}$.

The split feasibility problem (SFP) originally introduced by Censor and Elfving [1] is to find

$$
x \in C \text { such that } A x \in Q \text {, }
$$

where $A: H_{1} \longrightarrow H_{2}$ is a bounded linear operator. It serves as a model for many inverse problems where constraints are imposed on the solutions in the domain of a linear operator as well as in these operator's ranges. The applications of the split feasibility problem are very comprehensive such as CT in medicine, intelligence antennas, and the electronic warning systems in military, the development of fast image processing technology and HDTV, etc. Many authors generalize SFP to a lot of important problems, such as multiple-sets split feasibility problem, split equality fixed point problem, split variational inequality problem, split variational inclusion problem, and split equilibrium problem, and the theories and algorithms are studied and details can be seen in [2-15] and references therein.

The fixed point problem (FPP) for the mapping $T$ is to find $x \in C$ such that

$$
T x=x .
$$

We denote $\operatorname{Fix}(T):=\{x \in C: T x=x\}$ the set of solution of FPP.

Let $B: C \longrightarrow H_{1}$ be a nonlinear mapping. The variational inequality problem (VIP) is to find $x \in C$ such that

$$
\langle B x, y-x\rangle \geq 0, \quad \forall y \in C .
$$

The solution set of VIP is denoted by $\operatorname{VI}(C, B)$. It is well known that if $B$ is strongly monotone and Lipschitz continuous mapping on $C$, then VIP has a unique solution.

For finding a common problem of $\operatorname{Fix}(T) \cap \operatorname{VI}(C, B)$, Takahashi and Toyoda [16] introduced the following iterative scheme:

$$
\begin{gathered}
x_{0} \text { chosen arbitrary, } \\
x_{n+1}=\alpha_{n} x_{n}+\left(1-\alpha_{n}\right) T P_{C}\left(x_{n}-\lambda_{n} B x_{n}\right), \quad \forall n \geq 0,
\end{gathered}
$$


where $B$ is $\rho$-inverse-strongly monotone, $\left\{\alpha_{n}\right\}$ is a sequence in $(0,1)$, and $\left\{\lambda_{n}\right\}$ is a sequence in $(0,2 \rho)$. They showed that if $\operatorname{Fix}(T) \cap \operatorname{VI}(C, B) \neq \emptyset$, then the sequence $\left\{x_{n}\right\}$ generated by (4) converges weakly to $z_{0} \in \operatorname{Fix}(T) \cap \operatorname{VI}(C, B)$.

On the other hand, there are several numerical methods for solving variational inequalities and related optimization problems; see [5, 17-24] and the references therein.

In 2013, Kazmi and Rizvi [25] introduced the split generalized equilibrium problem (SGEP). Let $F_{1}, h_{1}: C \times$ $C \longrightarrow R$ and $F_{2}, h_{2}: Q \times Q \longrightarrow R$ be nonlinear bifunctions and $A: H_{1} \longrightarrow H_{2}$ be a bounded linear operator; then the split generalized equilibrium problem (SGEP) is to find $x^{*} \in C$ such that

$$
F_{1}\left(x^{*}, x\right)+h_{1}\left(x^{*}, x\right) \geq 0, \quad \forall x \in C
$$

and such that

$$
\begin{aligned}
y^{*}=A x^{*} \in Q \text { solves } F_{2}\left(y^{*}, y\right)+h_{2}\left(y^{*}, y\right) & \geq 0, \\
& \forall y \in Q .
\end{aligned}
$$

Denote the solution sets of generalized equilibrium problem (GEP) (5) and GEP (6) by $\operatorname{GEP}\left(F_{1}, h_{1}\right)$ and $\operatorname{GEP}\left(F_{2}, h_{2}\right)$, respectively. The solution set of SGEP is denoted by $\Gamma=$ $\left\{x^{*} \in \operatorname{GEP}\left(F_{1}, h_{1}\right): A x^{*} \in \operatorname{GEP}\left(F_{2}, h_{2}\right)\right\}$. They proposed the following iterative method for finding a common solution of split generalized equilibrium and fixed point problem. and

Let $\left\{x_{n}\right\}$ and $\left\{u_{n}\right\}$ be the sequences generated by $x_{0} \in C$

$$
\begin{aligned}
u_{n}= & T_{r_{n}}^{\left(F_{1}, h_{1}\right)}\left(x_{n}+\delta A^{*}\left(T_{r_{n}}^{\left(F_{2}, h_{2}\right)}-I\right) A x_{n}\right), \\
x_{n+1}= & \alpha_{n} \gamma f\left(x_{n}\right)+\beta_{n} x_{n} \\
& +\left(\left(1-\beta_{n}\right) I-\alpha_{n} D\right) \frac{1}{s_{n}} \int_{0}^{s_{n}} T(s) u_{n} d s,
\end{aligned}
$$

where $S=\{T(s): 0 \leq s<\infty\}$ is a nonexpansive semigroup on $C$ and $\operatorname{Fix}(S) \cap \Gamma \neq \emptyset, s_{n}$ is a positive real sequence which diverges to $+\infty,\left\{\alpha_{n}\right\},\left\{\beta_{n}\right\} \subset(0,1),\left\{r_{n}\right\} \subset(0, \infty)$, and $\delta \in$ $(0,1 / L), L$ is the spectral radius of the operator $A^{*} A, A^{*}$ is the adjoint of $A$, and

$$
\begin{aligned}
& T_{r}^{\left(F_{1}, h_{1}\right)}(x)=\left\{z \in C: F_{1}(z, y)+h_{1}(z, y)\right. \\
& \left.+\frac{1}{r}\langle y-z, z-x\rangle \geq 0, \forall y \in C\right\}, \\
& T_{s}^{\left(F_{2}, h_{2}\right)}(w)=\left\{d \in Q: F_{2}(d, e)+h_{2}(d, e)\right. \\
& \left.+\frac{1}{s}\langle e-d, d-w\rangle \geq 0, \forall e \in Q\right\} .
\end{aligned}
$$

Under suitable conditions, they proved a strong convergence theorem for the sequence generated by the proposed iterative scheme. But the calculation of integral is generally not easy. Therefore, it is necessary to reconsider the algorithm for solving this kind of problem.

Motivated by Kazmi and Rivi [25] as well as Che and Li [2], we introduce and study a kind of conjugate gradient viscosity approximation algorithm for finding a common solution of split generalized equilibrium problem and variational inequality problem. Under mild conditions, we prove that the sequence generated by the proposed iterative algorithm converges strongly to the common solution of $\mathrm{VI}(C, B)$ and SGEP. The results presented in this paper are the generalization, extension, and supplement of the previously known results in the corresponding references. Numerical results show the feasibility and efficiency of the proposed algorithm.

\section{Preliminaries}

In this section, we introduce some concepts and results which are needed in sequel.

A mapping $T: H_{1} \longrightarrow H_{1}$ is called

(1) contraction, if there exists a constant $\alpha \in(0,1)$ such that

$$
\|T x-T y\| \leq \alpha\|x-y\|, \quad \forall x, y \in H_{1} .
$$

If $\alpha=1$, then $T$ is called nonexpansive.

(2) monotone, if

$$
\langle T x-T y, x-y\rangle \geq 0, \quad \forall x, y \in H_{1} .
$$

(3) $\eta$-strongly monotone, if there exists a positive constant $\eta$ such that

$$
\langle T x-T y, x-y\rangle \geq \eta\|x-y\|^{2}, \quad \forall x, y \in H_{1} .
$$

(4) $\alpha$-inverse strongly monotone ( $\alpha$-ism), if there exists a constant $\alpha>0$ such that

$$
\langle T x-T y, x-y\rangle \geq \alpha\|T x-T y\|^{2}, \quad \forall x, y \in H_{1} .
$$

(5) firmly nonexpansive, if

$$
\langle T x-T y, x-y\rangle \geq\|T x-T y\|^{2}, \quad \forall x, y \in H_{1} .
$$

A mapping $P_{C}$ is said to be metric projection of $H_{1}$ onto $C$ if, for every point $x \in H_{1}$, there exists a unique nearest point in $C$ denoted by $P_{C} x$ such that

$$
\left\|x-P_{C} x\right\| \leq\|x-y\|, \quad \forall y \in C .
$$

It is well known that $P_{C}$ is a nonexpansive mapping and is characterized by the following properties:

$$
\begin{gathered}
\left\|P_{C} x-P_{C} y\right\|^{2} \leq\left\langle x-y, P_{C} x-P_{C} y\right\rangle, \\
\forall x, y \in H_{1}, \\
\left\langle x-P_{C} x, y-P_{C} x\right\rangle \leq 0, \quad \forall x \in H_{1}, \quad y \in C, \\
\|x-y\|^{2} \geq\left\|x-P_{C} x\right\|^{2}+\left\|y-P_{C} x\right\|^{2}, \\
\forall x \in H_{1}, \quad y \in C
\end{gathered}
$$

and

$$
\begin{aligned}
& \left\|(x-y)-\left(P_{C} x-P_{C} y\right)\right\|^{2} \\
& \quad \geq\|x-y\|^{2}-\left\|P_{C} x-P_{C} y\right\|^{2}, \quad \forall x, y \in H_{1} .
\end{aligned}
$$


A linear bounded operator $B$ is strongly positive if there exists a constant $\bar{\gamma}>0$ with the property

$$
\langle B x, x\rangle \geq \bar{\gamma}\|x\|^{2}, \quad \forall x \in H_{1} .
$$

A mapping $T: H_{1} \longrightarrow H_{1}$ is said to be averaged if and only if it can be written as the average of the identity mapping and a nonexpansive mapping; i.e.,

$$
T=(1-\alpha) I+\alpha S,
$$

where $\alpha \in(0,1), S: H_{1} \longrightarrow H_{1}$ is nonexpansive, and $I$ is the identity operator on $H_{1}$. More precisely, we say that $T$ is $\alpha$-averaged. We note that averaged mapping is nonexpansive. Furthermore, firmly nonexpansive mapping (in particular, projection on nonempty closed and convex subset) is averaged.

Let $B$ be a monotone mapping of $C$ into $H_{1}$. In the context of the variational inequality problem, the characterization of projection (17) implies the following relation:

$$
u \in \mathrm{VI}(C, B) \Longleftrightarrow u=P_{C}(u-\lambda B u), \quad \lambda>0 .
$$

In the proof of our results, we need the following assumptions and lemmas.

Lemma 1 (see [26]). If $x, y, z \in H_{1}$, then

(i) $\|x+y\|^{2} \leq\|x\|^{2}+2\langle y, x+y\rangle$.

(ii) For any $\lambda \in[0,1]$,

$$
\begin{aligned}
\|\lambda x+(1-\lambda) y\|^{2}= & \lambda\|x\|^{2}+(1-\lambda)\|y\|^{2} \\
& -\lambda(1-\lambda)\|x-y\|^{2} .
\end{aligned}
$$

(iii) For $a, b, c \in[0,1]$ with $a+b+c=1$,

$$
\begin{aligned}
\|a x+b y+c z\|^{2}= & a\|x\|^{2}+b\|y\|^{2}+c\|z\|^{2} \\
& -a b\|x-y\|^{2}-a c\|x-z\|^{2} \\
& -b c\|y-z\|^{2} .
\end{aligned}
$$

Assumption 2. Let $F: C \times C \longrightarrow R$ be a bifunction satisfying the following assumption:

(i) $F(x, x) \geq 0, \forall x \in C$.

(ii) $F$ is monotone; i.e., $F(x, y)+F(y, x) \leq 0, \forall x \in C$.

(iii) $F$ is upper hemicontinuous; i.e., for each $x, y, z \in C$,

$$
\limsup _{t \rightarrow 0} F(t z+(1-t) x, y) \leq F(x, y) .
$$

(iv) For each $x \in C$ fixed, the function $y \longmapsto F(x, y)$ is convex and lower semicontinuous.

Let $h: C \times C \longrightarrow R$ such that

(i) $h(x, x) \geq 0, \forall x \in C$

(ii) for each $y \in C$ fixed, the function $x \longrightarrow h(x, y)$ is upper semicontinuous

(iii) for each $x \in C$ fixed, the function $y \longrightarrow h(x, y)$ is convex and lower semicontinuous
And assume that, for fixed $r>0$ and $z \in C$, there exists a nonempty compact convex subset $K$ of $H_{1}$ and $x \in C \cap K$ such that

$$
F(y, x)+h(y, x)+\frac{1}{r}\langle y-x, x-z\rangle<0,
$$

$$
\forall y \in C \backslash K \text {. }
$$

Lemma 3 (see [25]). Assume that $F_{1}, h_{1}: C \times C \longrightarrow R$ satisfy Assumption 2. Let $r>0$ and $x \in H_{1}$. Then, there exists $z \in C$ such that

$$
F_{1}(z, y)+h_{1}(z, y)+\frac{1}{r}\langle y-z, z-x\rangle \geq 0,
$$

$\forall y \in C$.

Lemma 4 (see [1]). Assume that the bifunctions $F_{1}, h_{1}: C \times$ $C \longrightarrow R$ satisfy Assumption 2 and $h_{1}$ is monotone. For $r>0$ and for all $x \in H_{1}$, define a mapping $T_{r}^{\left(F_{1}, h_{1}\right)}: H_{1} \longrightarrow C$ as (8). Then, the following conclusions hold.

(1) $T_{r}^{\left(F_{1}, h_{1}\right)}$ is single-valued.

(2) $T_{r}^{\left(F_{1}, h_{1}\right)}$ is firmly nonexpansive.

(3) $\operatorname{Fix}\left(T_{r}^{\left(F_{1}, h_{1}\right)}\right)=\operatorname{GEP}\left(F_{1}, h_{1}\right)$.

(4) $\operatorname{GEP}\left(F_{1}, h_{1}\right)$ is compact and convex.

Furthermore, assume that $F_{2}, h_{2}: Q \times Q \longrightarrow R$ satisfy Assumption 2. For $s>0$ and, for all $w \in H_{2}, T_{s}^{\left(F_{2}, h_{2}\right)}: H_{2} \longrightarrow$ $Q$ is defined as (9). By Lemma 4, we easily observe that $T_{s}^{\left(F_{2}, h_{2}\right)}$ is single-valued and firmly nonexpansive, $\operatorname{GEP}\left(F_{2}, h_{2}, Q\right)$ is compact and convex, and $\operatorname{Fix}\left(T_{s}^{\left(F_{2}, h_{2}\right)}\right)=\operatorname{GEP}\left(F_{2}, h_{2}, Q\right)$, where $\operatorname{GEP}\left(F_{2}, h_{2}, Q\right)$ is the solution set of the following generalized equilibrium problem, which is to find $y^{*} \in Q$ such that $F_{2}\left(y^{*}, y\right)+h_{2}\left(y^{*}, y\right) \geq 0, \forall y \in Q$.

We observe that $\operatorname{GEP}\left(F_{2}, h_{2}\right) \subset \operatorname{GEP}\left(F_{2}, h_{2}, Q\right)$. Further, it is easy to prove that $\Gamma$ is a closed and convex set.

Lemma 5 (see $[27,28]$ ). Assume that $T: H_{1} \longrightarrow H_{1}$ is nonexpansive operator. For all $(x, y) \in H_{1} \times H_{1}$, the following inequality is true

$$
\begin{aligned}
& \langle(x-T(x))-(y-T(y)), T(y)-T(x)\rangle \\
& \quad \leq \frac{1}{2}\|(T(x)-x)-(T(y)-y)\|^{2} .
\end{aligned}
$$

And for all $(x, y) \in H_{1} \times$ Fix $(T)$, one has

$$
\langle x-T(x), y-T(x)\rangle \leq \frac{1}{2}\|T(x)-x\|^{2} .
$$

Lemma 6 (see [29]). Assume $A$ is a strongly positive linear bounded operator on Hilbert space $H_{1}$ with coefficient $\bar{\gamma}>0$ and $0<\rho \leq\|A\|^{-1}$. Then $\|I-\rho A\| \leq 1-\rho \bar{\gamma}$.

Lemma 7 (see $[30,31]$ ). Assume that $\left\{a_{n}\right\}$ is a sequence of nonnegative real numbers such that

$$
a_{n+1} \leq\left(1-\gamma_{n}\right) a_{n}+\gamma_{n} \delta_{n}+\beta_{n}, \quad n \geq 0,
$$

where $\left\{\gamma_{n}\right\}$ and $\left\{\beta_{n}\right\}$ are sequences in $(0,1)$ and $\left\{\delta_{n}\right\}$ is a sequence in $R$, such that 
(i) $\sum_{n=0}^{\infty} \gamma_{n}=\infty$.

(ii) $\limsup _{n \rightarrow \infty} \delta_{n} \leq 0$ or $\sum_{n=0}^{\infty}\left|\gamma_{n} \delta_{n}\right|<\infty$.

(iii) $\sum_{n=0}^{\infty} \beta_{n}<\infty$.

Then $\lim _{n \rightarrow \infty} a_{n}=0$.

According to [28], it is easy to prove the following Lemma.

Lemma 8. Let $F_{1}, h_{1}: C \times C \longrightarrow R$ be bifunctions satisfying Assumption 2 and, for $r>0$, the mapping $T_{r}^{\left(F_{1}, h_{1}\right)}$ is defined as (8). Let $x, y \in H_{1}$, and $r_{1}, r_{2}>0$. Then

$$
\begin{aligned}
\left\|T_{r_{2}}^{\left(F_{1}, h_{1}\right)} y-T_{r_{1}}^{\left(F_{1}, h_{1}\right)} x\right\| \leq & \|y-x\| \\
& +\left|\frac{r_{2}-r_{1}}{r_{2}}\right|\left\|T_{r_{2}}^{\left(F_{1}, h_{1}\right)} y-y\right\| .
\end{aligned}
$$

Lemma 9 (see $[32,33]$ ). Let $T: H \longrightarrow H$ be given. We have the following:

(i) $T$ is nonexpansive, iff the complement $I-T$ is $1 / 2$-ism

(ii) if $T$ is $v$-ism, then, for $\gamma>0, \gamma T$ is $v / \gamma$-ism

(iii) $T$ is averaged, iff the complement $I-T$ is $v$-ism for some $v>1 / 2$; indeed, for $\alpha \in(0,1), T$ is $\alpha$-averaged, iff $I-T$ is $1 / 2 \alpha$-ism

Lemma 10 (see $[4,32]$ ). Let the operators $S, T, V: H \longrightarrow H$ be given.

(i) If $T=(1-\alpha) S+\alpha V$, where $S$ is averaged, $V$ is nonexpansive, and $\alpha \in(0,1)$, then $T$ is averaged.

(ii) $T$ is firmly nonexpansive, iff the complement $I-T$ is firmly nonexpansive.

(iii) If $T=(1-\alpha) S+\alpha V$, where $S$ is firmly nonexpansive, $V$ is nonexpansive, and $\alpha \in(0,1)$, then $T$ is averaged.

(iv) The composite of finitely many averaged mappings is averaged. That is, if each of the mappings $\left\{T_{i}\right\}_{i=1}^{N}$ is averaged, then so is the composite $T_{1}, \ldots, T_{N}$. In particular, if $T_{1}$ is $\alpha_{1}$ averaged and $T_{2}$ is $\alpha_{2}$-averaged, where $\alpha_{1}, \alpha_{2} \in(0,1)$, then the composite $T_{1} T_{2}$ is $\alpha$-averaged, where $\alpha=\alpha_{1}+\alpha_{2}-\alpha_{1} \alpha_{2}$.

(v) If the mappings $\left\{T_{i}\right\}_{i=1}^{N}$ are averaged and have a common fixed point, then

$$
\bigcap_{i=1}^{N} \operatorname{Fix}\left(T_{i}\right)=\operatorname{Fix}\left(T_{1}, \ldots, T_{N}\right) .
$$

In the following, we give the relation between the projection operator and average mapping.

Lemma 11. Assume that the variational inequality problem (3) is solvable. If $B$ is $\beta$-ism from $C$ into $H_{1}$, then $P_{C}(I-\lambda B)$ is $(2 \beta+\lambda) / 4 \beta$-averaged.

Proof. Note that $B$ is $\beta$-ism, which implies that $\lambda B$ is $\beta / \lambda$-ism; i.e., $I-(I-\lambda B)$ is $\beta / \lambda$-ism. By Lemma 9(iii), we can see that $I-$ $\lambda B$ is $\lambda / 2 \beta$-averaged. Since the projection $P_{C}$ is $1 / 2$-averaged, it is easy to see from Lemma 10 that the composite $P_{C}(I-\lambda B)$ is $(2 \beta+\lambda) / 4 \beta$-averaged for $0<\lambda<2 \beta$ according to

$$
\frac{1}{2}+\frac{\lambda}{2 \beta}-\frac{1}{2} \cdot \frac{\lambda}{2 \beta}=\frac{2 \beta+\lambda}{4 \beta}
$$

which completes the proof.
As a result we have that, for each $n, P_{C}\left(I-\lambda_{n} B\right)$ is $(2 \beta+$ $\left.\lambda_{n}\right) / 4 \beta$-averaged. Therefore, we can write

$$
\begin{aligned}
P_{C}\left(I-\lambda_{n} B\right) & =\frac{2 \beta-\lambda_{n}}{4 \beta} I+\frac{2 \beta+\lambda_{n}}{4 \beta} T_{n} \\
& =\left(1-b_{n}\right) I+b_{n} T_{n},
\end{aligned}
$$

where $T_{n}$ is nonexpansive and $b_{n}=\left(2 \beta+\lambda_{n}\right) / 4 \beta \in[1 / 2,1]$.

Lemma 12 (see [28]). Let $\left\{x_{n}\right\}$ and $\left\{z_{n}\right\}$ be bounded sequence in a Banach space $X$ and let $\left\{\beta_{n}\right\}$ be a sequence in $[0,1]$ with $0<\liminf _{n \rightarrow \infty} \beta_{n} \leq \limsup _{n \rightarrow \infty} \beta_{n}<1$. Suppose $x_{n+1}=$ $\left(1-\beta_{n}\right) z_{n}+\beta_{n} x_{n}$ for all integers $n \geq 0$ and lim $\sup _{n \rightarrow \infty}\left(\| z_{n+1}-\right.$ $\left.z_{n}\|-\| x_{n+1}-x_{n} \|\right) \leq 0$. Then $\lim _{n \rightarrow \infty}\left\|z_{n}-x_{n}\right\|=0$.

\section{Main Results}

In this section, we give the main results of this paper. First, we describe the algorithm for finding a common solution of split generalized equilibrium and variational inequality problems.

Throughout the rest of this paper, let $f$ be a contraction of $H_{1}$ into itself with coefficient $\eta \in(0,1), A$ be a bounded linear operator, $B$ be a $\beta$-inverse strongly monotone mapping from $C$ into $H_{1}$, and $D$ be a strongly positive linear bounded selfadjoint operator on $H_{1}$ with coefficient $\bar{\gamma}>0$ and $0<\gamma<\bar{\gamma} / \eta$.

Now, we give the description of the algorithm.

Algorithm 13. Let $x_{0} \in H_{1}$ be arbitrary and $\alpha>0$. Assume that $\left\{\alpha_{n}\right\},\left\{\beta_{n}\right\},\left\{\gamma_{n}\right\} \subset(0,1),\left\{\lambda_{n}\right\} \subset(0,2 \beta),\left\{r_{n}\right\} \subset(0, \infty)$, and $\xi \in(0,1 / L)$, where $L$ is the spectral radius of the operator $A A^{*}$ and $A^{*}$ is the adjoint of $A$. Calculate sequences $\left\{u_{n}\right\}$, $\left\{y_{n}\right\}$, and $\left\{x_{n}\right\}$ by the following iteration formula.

$$
\begin{aligned}
u_{n} & =T_{r_{n}}^{\left(F_{1}, h_{1}\right)}\left(x_{n}+\xi A^{*}\left(T_{r_{n}}^{\left(F_{2}, h_{2}\right)}-I\right) A x_{n}\right), \\
y_{n} & =u_{n}+\alpha d_{n+1}, \\
x_{n+1} & =\alpha_{n} \gamma f\left(x_{n}\right)+\beta_{n} x_{n}+\left(\left(1-\beta_{n}\right) I-\alpha_{n} D\right) y_{n},
\end{aligned}
$$

where $d_{n+1}=(1 / \alpha)\left(T_{n} u_{n}-u_{n}\right)+\gamma_{n} d_{n}, d_{0}=(1 / \alpha)\left(T_{0} u_{0}-u_{0}\right)$ and $T_{n}$ is defined by (34).

As follows, we propose the convergence analysis of Algorithm 13.

Theorem 14. Let $H_{1}$ and $H_{2}$ be two real Hilbert spaces and $C \mathrm{C}$ $H_{1}, Q \subset H_{2}$ be nonempty closed convex subsets. Let $F_{1}, h_{1}$ : $C \times C \longrightarrow R$ and $F_{2}, h_{2}: Q \times Q \longrightarrow R$ satisfy Assumption 2; $h_{1}, h_{2}$ are monotone and $F_{2}$ is upper semicontinuous in the first argument. Assume that $\Omega:=V I(C, B) \cap \Gamma \neq \emptyset$ and $\left\{x_{n}\right\},\left\{u_{n}\right\}$, and $\left\{y_{n}\right\}$ are generated by (35). Suppose that $\left\{\alpha_{n}\right\},\left\{\beta_{n}\right\} \subset(0,1)$, $\left\{\gamma_{n}\right\} \subset(0,1 / 2),\left\{\lambda_{n}\right\} \subset(0,2 \beta),\left\{r_{n}\right\} \subset(0, \infty)$ satisfy the following conditions:

(C1) $\lim _{n \rightarrow \infty} \alpha_{n}=0, \sum_{n=0}^{\infty} \alpha_{n}=\infty$.

(C2) $0<\liminf _{n \rightarrow \infty} \beta_{n} \leq \lim \sup _{n \rightarrow \infty} \beta_{n}<1$.

(C3) $\gamma_{n}=o\left(\alpha_{n}\right)$.

(C4) $\liminf _{n \rightarrow \infty} \lambda_{n}>0, \lim _{n \rightarrow \infty}\left|\lambda_{n+1}-\lambda_{n}\right|=0$.

(C5) $\lim _{n \rightarrow \infty}\left(\left|r_{n+1}-r_{n}\right| / r_{n+1}\right)=0$.

(C6) $\left\{T_{n} u_{n}-u_{n}\right\}$ is bounded. 
Then the sequence $\left\{x_{n}\right\}$ converges strongly to $q \in \Omega$, where $q=P_{\Omega}(I-D+\gamma f) q$, which is the unique solution of the variational inequality problem

$$
\langle(D-\gamma f) q, x-q\rangle \geq 0, \quad \forall x \in \Omega,
$$

or equivalently, $q$ is the unique solution to the minimization problem

$$
\min _{x \in \Omega} \frac{1}{2}\langle D x, x\rangle-h(x),
$$

where $h$ is a potential function for $\gamma f$ such that $h^{\prime}(x)=\gamma f(x)$ for $x \in H_{1}$.

Proof. Some equalities and inequalities in the following can be obtained according to the proof of Theorem 1 in [25]. However, we give the detailed proof process in order to read handily.

From $(\mathrm{Cl})$ and $(\mathrm{C} 2)$, without loss of generality we assume that $\alpha_{n} \leq\left(1-\beta_{n}\right)\|D\|^{-1}$ for all $n \in N$. By Lemma 6, we have $\|I-\rho D\| \leq 1-\rho \bar{\gamma}$ if $0<\rho \leq\|D\|^{-1}$. Now suppose that $\|I-D\| \leq 1-\bar{\gamma}$. Since $D$ is a strongly positive linear bounded self-adjoint operator on $H_{1}$, we obtain

$$
\|D\|=\sup \left\{|\langle D x, x\rangle|: x \in H_{1},\|x\|=1\right\} .
$$

Notice that

$$
\begin{aligned}
& \left\langle\left(\left(1-\beta_{n}\right) I-\alpha_{n} D\right) x, x\right\rangle=1-\beta_{n}-\alpha_{n}\langle D x, x\rangle \\
& \quad \geq 1-\beta_{n}-\alpha_{n}\|D\| \geq 0,
\end{aligned}
$$

which shows that $\left(1-\beta_{n}\right) I-\alpha_{n} D$ is positive definite. Furthermore, we have

$$
\begin{aligned}
\|( & \left.-\beta_{n}\right) I-\alpha_{n} D \| \\
& =\sup \left\{\left|\left\langle\left(\left(1-\beta_{n}\right) I-\alpha_{n} D\right) x, x\right\rangle\right|: x \in H_{1},\|x\|\right. \\
& =1\}=\sup \left\{1-\beta_{n}-\alpha_{n}\langle D x, x\rangle: x \in H_{1},\|x\|=1\right\} \\
& \leq 1-\beta_{n}-\alpha_{n} \bar{\gamma} .
\end{aligned}
$$

Since $f$ is a contraction mapping with constant $\eta \in(0,1)$, for all $x, y \in H_{1}$, we have

$$
\begin{aligned}
& \left\|P_{\Omega}(I-D+\gamma f)(x)-P_{\Omega}(I-D+\gamma f)(y)\right\| \\
& \quad \leq\|(I-D+\gamma f)(x)-(I-D+\gamma f)(y)\| \\
& \quad \leq\|I-D\|\|x-y\|+\gamma\|f(x)-f(y)\| \\
& \quad \leq(1-\bar{\gamma})\|x-y\|+\gamma \eta\|x-y\| \\
& \quad \leq(1-(\bar{\gamma}-\gamma \eta))\|x-y\|,
\end{aligned}
$$

which implies that $P_{\Omega}(I-D+\gamma f)$ is a contraction mapping from $H_{1}$ into itself. It follows from the Banach contraction principle that there exists an element $q \in \Omega$ such that $q=$ $P_{\Omega}(I-D+\gamma f) q$.

Step 1 (we show that $\left\{x_{n}\right\}$ is bounded). Let $x^{*} \in \Omega$; i.e., $x^{*} \in \Gamma$; we have $x^{*}=T_{r_{n}}^{\left(F_{1}, h_{1}\right)} x^{*}$ and $A x^{*}=T_{r_{n}}^{\left(F_{2}, h_{2}\right)} A x^{*}$.
In the following, we compute

$$
\begin{aligned}
& \left\|u_{n}-x^{*}\right\|^{2}=\| T_{r_{n}}^{\left(F_{1}, h_{1}\right)}\left(x_{n}+\xi A^{*}\left(T_{r_{n}}^{\left(F_{2}, h_{2}\right)}-I\right) A x_{n}\right) \\
& -x^{*}\left\|^{2}=\right\| T_{r_{n}}^{\left(F_{1}, h_{1}\right)}\left(x_{n}+\xi A^{*}\left(T_{r_{n}}^{\left(F_{2}, h_{2}\right)}-I\right) A x_{n}\right) \\
& -T_{r_{n}}^{\left(F_{1}, h_{1}\right)} x^{*}\left\|^{2} \leq\right\| x_{n}+\xi A^{*}\left(T_{r_{n}}^{\left(F_{2}, h_{2}\right)}-I\right) A x_{n} \\
& -x^{*}\left\|^{2}=\right\| x_{n}-x^{*}\left\|^{2}+\xi^{2}\right\| A^{*}\left(T_{r_{n}}^{\left(F_{2}, h_{2}\right)}-I\right) A x_{n} \|^{2} \\
& +2 \xi\left\langle x_{n}-x^{*}, A^{*}\left(T_{r_{n}}^{\left(F_{2}, h_{2}\right)}-I\right) A x_{n}\right\rangle .
\end{aligned}
$$

Thus, we have

$$
\begin{aligned}
& \left\|u_{n}-x^{*}\right\|^{2} \\
& \leq\left\|x_{n}-x^{*}\right\|^{2} \\
& \quad+\xi^{2}\left\langle\left(T_{r_{n}}^{\left(F_{2}, h_{2}\right)}-I\right) A x_{n}, A A^{*}\left(T_{r_{n}}^{\left(F_{2}, h_{2}\right)}-I\right) A x_{n}\right\rangle \\
& \quad+2 \xi\left\langle x_{n}-x^{*}, A^{*}\left(T_{r_{n}}^{\left(F_{2}, h_{2}\right)}-I\right) A x_{n}\right\rangle .
\end{aligned}
$$

On the other hand, we have

$$
\begin{aligned}
\xi^{2} & \left\langle\left(T_{r_{n}}^{\left(F_{2}, h_{2}\right)}-I\right) A x_{n}, A A^{*}\left(T_{r_{n}}^{\left(F_{2}, h_{2}\right)}-I\right) A x_{n}\right\rangle \\
& \leq L \xi^{2}\left\|\left(T_{r_{n}}^{\left(F_{2}, h_{2}\right)}-I\right) A x_{n}\right\|^{2} .
\end{aligned}
$$

and

$$
\begin{aligned}
2 \xi & \left\langle x_{n}-x^{*}, A^{*}\left(T_{r_{n}}^{\left(F_{2}, h_{2}\right)}-I\right) A x_{n}\right\rangle \\
& =2 \xi\left\langle A\left(x_{n}-x^{*}\right),\left(T_{r_{n}}^{\left(F_{2}, h_{2}\right)}-I\right) A x_{n}\right\rangle \\
& =2 \xi\left\langle A\left(x_{n}-x^{*}\right)+\left(T_{r_{n}}^{\left(F_{2}, h_{2}\right)}-I\right) A x_{n}\right. \\
& \left.-\left(T_{r_{n}}^{\left(F_{2}, h_{2}\right)}-I\right) A x_{n},\left(T_{r_{n}}^{\left(F_{2}, h_{2}\right)}-I\right) A x_{n}\right\rangle \\
& =2 \xi\left\{\left\langle T_{\left.r_{n}, h_{2}\right)}^{\left(F_{2}, h_{n}\right)}-A x^{*},\left(T_{r_{n}}^{\left(F_{2}, h_{2}\right)}-I\right) A x_{n}\right\rangle\right. \\
& \left.-\left\|\left(T_{r_{n}}^{\left(F_{2}, h_{2}\right)}-I\right) A x_{n}\right\|^{2}\right\} \\
& \leq 2 \xi\left\{\frac{1}{2}\left\|\left(T_{r_{n}}^{\left(F_{2}, h_{2}\right)}-I\right) A x_{n}\right\|^{2}\right. \\
& \left.-\left\|\left(T_{r_{n}}^{\left(F_{2}, h_{2}\right)}-I\right) A x_{n}\right\|^{2}\right\} \\
& \leq-\xi\left\|\left(T_{r_{n}}^{\left(F_{2}, h_{2}\right)}-I\right) A x_{n}\right\|^{2},
\end{aligned}
$$

where the first inequality is derived from (29).

From (43)-(45), we have

$$
\begin{aligned}
\left\|u_{n}-x^{*}\right\|^{2} \leq & \left\|x_{n}-x^{*}\right\|^{2} \\
& +\xi(L \xi-1)\left\|\left(T_{r_{n}}^{\left(F_{2}, h_{2}\right)}-I\right) A x_{n}\right\|^{2} .
\end{aligned}
$$

Noticing that $\xi \in(0,1 / L)$, we obtain

$$
\left\|u_{n}-x^{*}\right\|^{2} \leq\left\|x_{n}-x^{*}\right\|^{2} .
$$


As follows, we prove that $\left\{d_{n}\right\}$ is bounded. The proof is by induction. It is true trivially for $n=0$. Let $M_{1}=$ $\max \left\{\left\|d_{0}\right\|,(2 / \alpha) \sup _{n \in N}\left\|T_{n} u_{n}-u_{n}\right\|\right\}$. From (C6), it is shown that $M_{1}<\infty$. Assume that $\left\|d_{n}\right\| \leq M_{1}$ for some $n$; we prove that it holds for $n+1$. According to the triangle inequality, we obtain

$$
\begin{aligned}
\left\|d_{n+1}\right\| & =\left\|\frac{1}{\alpha}\left(T_{n} u_{n}-u_{n}\right)+\gamma_{n} d_{n}\right\| \\
& \leq \frac{1}{\alpha}\left\|T_{n} u_{n}-u_{n}\right\|+\gamma_{n}\left\|d_{n}\right\| \leq \frac{1}{\alpha} \cdot \frac{\alpha}{2} M_{1}+\frac{M_{1}}{2} \\
& =M_{1},
\end{aligned}
$$

which implies that $\left\|d_{n}\right\| \leq M_{1}$ for all $n \in N$; i.e., $\left\{d_{n}\right\}$ is bounded.

It is easy to see that $x^{*} \in V I(C, B)$ according to $x^{*} \in \Omega$. By (22), we have $P_{C}(I-\lambda B) x^{*}=x^{*}$, which, together with (34), implies that

$$
b_{n} T_{n} x^{*}=P_{C}(I-\lambda B) x^{*}-\left(1-b_{n}\right) x^{*}=b_{n} x^{*} ;
$$

that is,

$$
T_{n} x^{*}=x^{*} .
$$

By the definition of $\left\{y_{n}\right\}$, (47), and $\left\{T_{n}\right\}$ being nonexpansive, we have

$$
\begin{aligned}
\left\|y_{n}-x^{*}\right\| & =\left\|u_{n}+\alpha d_{n+1}-x^{*}\right\| \\
& =\left\|T_{n}\left(u_{n}\right)+\alpha \gamma_{n} d_{n}-T_{n} x^{*}\right\| \\
& \leq\left\|u_{n}-x^{*}\right\|+\alpha \gamma_{n} M_{1} \\
& \leq\left\|x_{n}-x^{*}\right\|+\alpha \gamma_{n} M_{1} .
\end{aligned}
$$

As a result, it follows from (51), Lemma 6, and the fact that $\alpha_{n} \longrightarrow 0$ and $\gamma_{n}=o\left(\alpha_{n}\right)$ that when $n$ is large enough,

$$
\begin{gathered}
\left\|x_{n+1}-x^{*}\right\|=\| \alpha_{n}\left(\gamma f\left(x_{n}\right)-D x^{*}\right)+\beta_{n}\left(x_{n}-x^{*}\right) \\
+\left(\left(1-\beta_{n}\right) I-\alpha_{n} D\right)\left(y_{n}-x^{*}\right)\left\|\leq \alpha_{n}\right\| \gamma f\left(x_{n}\right) \\
-D x^{*}\left\|+\beta_{n}\right\| x_{n}-x^{*} \|+\left(1-\beta_{n}-\alpha_{n} \bar{\gamma}\right) \\
\cdot\left(\left\|x_{n}-x^{*}\right\|+\alpha \gamma_{n} M_{1}\right) \leq \alpha_{n} \gamma\left\|f\left(x_{n}\right)-f\left(x^{*}\right)\right\| \\
+\alpha_{n}\left\|\gamma f\left(x^{*}\right)-D x^{*}\right\|+\beta_{n}\left\|x_{n}-x^{*}\right\|+\left(1-\beta_{n}\right. \\
\left.-\alpha_{n} \bar{\gamma}\right)\left(\left\|x_{n}-x^{*}\right\|+\alpha \gamma_{n} M_{1}\right) \leq \alpha_{n} \gamma \eta\left\|x_{n}-x^{*}\right\| \\
+\alpha_{n}\left\|\gamma f\left(x^{*}\right)-D x^{*}\right\|+\left(1-\alpha_{n} \bar{\gamma}\right)\left\|x_{n}-x^{*}\right\|+(1 \\
\left.-\beta_{n}-\alpha_{n} \bar{\gamma}\right) \alpha \gamma_{n} M_{1} \leq\left(1-\alpha_{n}(\bar{\gamma}-\gamma \eta)\right)\left\|x_{n}-x^{*}\right\| \\
+\alpha_{n}(\bar{\gamma}-\gamma \eta) \frac{\alpha M_{1}+\left\|\gamma f\left(x^{*}\right)-D x^{*}\right\|}{\bar{\gamma}-\gamma \eta} \\
\leq \max \left\{\left\|x_{n}-x^{*}\right\|, \frac{\alpha M_{1}+\left\|\gamma f\left(x^{*}\right)-D x^{*}\right\|}{\bar{\gamma}-\gamma \eta}\right\},
\end{gathered}
$$

where the third inequality is true because $\beta_{n} \in(0,1), \alpha_{n} \longrightarrow$ 0 , and $\gamma_{n}=o\left(\alpha_{n}\right)$. As a result,

$$
\left(1-\beta_{n}-\alpha_{n} \bar{\gamma}\right) \alpha \gamma_{n} M_{1} \leq \alpha \alpha_{n} M_{1},
$$

when $n$ is large enough.

Hence, $\left\{x_{n}\right\}$ is bounded and so are $\left\{u_{n}\right\}$ and $\left\{y_{n}\right\}$.

Step 2 (we show that $\lim _{n \rightarrow \infty}\left\|x_{n+1}-x_{n}\right\|=0$ ). Since $T_{r_{n+1}}^{\left(F_{1}, h_{1}\right)}$ and $T_{r_{n+1}}^{\left(F_{2}, h_{2}\right)}$ both are firmly nonexpansive, for $\xi \in(0,1 / L)$, the mapping $T_{r_{n+1}}^{\left(F_{1}, h_{1}\right)}\left(I+\xi A^{*}\left(T_{r_{n+1}}^{\left(F_{2}, h_{2}\right)}-I\right) A\right)$ is nonexpansive; see [34, 35]. Noticing that $u_{n}=T_{r_{n}}^{\left(F_{1}, h_{1}\right)}\left(x_{n}+\xi A^{*}\left(T_{r_{n}}^{\left(F_{2}, h_{2}\right)}-I\right) A x_{n}\right)$ and $u_{n+1}=T_{r_{n+1}}^{\left(F_{1}, h_{1}\right)}\left(x_{n+1}+\xi A^{*}\left(T_{r_{n+1}}^{\left(F_{2}, h_{2}\right)}-I\right) A x_{n+1}\right)$, we have from Lemma 8 that

$$
\begin{aligned}
& \left\|u_{n+1}-u_{n}\right\| \\
& \quad \leq \| T_{r_{n+1}}^{\left(F_{1}, h_{1}\right)}\left(x_{n+1}+\xi A^{*}\left(T_{r_{n+1}}^{\left(F_{2}, h_{2}\right)}-I\right) A x_{n+1}\right) \\
& \quad-T_{r_{n+1}}^{\left(F_{1}, h_{1}\right)}\left(x_{n}+\xi A^{*}\left(T_{r_{n+1}}^{\left(F_{2}, h_{2}\right)}-I\right) A x_{n}\right) \| \\
& \quad+\| T_{r_{n+1}}^{\left(F_{1}, h_{1}\right)}\left(x_{n}+\xi A^{*}\left(T_{r_{n+1}}^{\left(F_{2}, h_{2}\right)}-I\right) A x_{n}\right) \\
& \quad-T_{r_{n}}^{\left(F_{1}, h_{1}\right)}\left(x_{n}+\xi A^{*}\left(T_{r_{n}}^{\left(F_{2}, h_{2}\right)}-I\right) A x_{n}\right)\|\leq\| x_{n+1} \\
& -x_{n}\|+\| x_{n}+\xi A^{*}\left(T_{r_{n+1}}^{\left(F_{2}, h_{2}\right)}-I\right) A x_{n} \\
& \quad-\left(x_{n}+\xi A^{*}\left(T_{r_{n}}^{\left(F_{2}, h_{2}\right)}-I\right) A x_{n}\right) \|+\left|1-\frac{r_{n}}{r_{n+1}}\right| \\
& \quad \| T_{r_{n+1}}^{\left(F_{1}, h_{1}\right)}\left(x_{n}+\xi A^{*}\left(T_{r_{n+1}}^{\left(F_{2}, h_{2}\right)}-I\right) A x_{n}\right) \\
& -\left(x_{n}+\xi A^{*}\left(T_{r_{n+1}}^{\left(F_{2}, h_{2}\right)}-I\right) A x_{n}\right)\|\leq\| x_{n+1}-x_{n} \| \\
& \quad+\xi\|A\|\left\|T_{r_{n+1}}^{\left(F_{2}, h_{2}\right)} A x_{n}-T_{r_{n}}^{\left(F_{2}, h_{2}\right)} A x_{n}\right\|+\zeta_{n} \leq \| x_{n+1} \\
& \quad-x_{n}\|+\xi\| A\left\|\left|1-\frac{r_{n}}{r_{n+1}}\right|\right\| T_{r_{n+1}}^{\left(F_{2}, h_{2}\right)} A x_{n}-A x_{n} \|+\zeta_{n} \\
& \quad=\left\|x_{n+1}-x_{n}\right\|+\xi\|A\| \sigma_{n}+\zeta_{n},
\end{aligned}
$$

where $\sigma_{n}:=\left|1-r_{n} / r_{n+1}\right| \mid T_{r_{2}}^{\left(F_{2}, h_{2}\right)} A x_{n}-A x_{n} \|$ and $\zeta_{n}:=\mid 1-$ $r_{n} / r_{n+1} \mid \| T_{r_{n+1}}^{\left(F_{1}, h_{1}\right)}\left(x_{n}+\xi A^{*}\left(T_{r_{n}}^{\left(F_{2}, h_{2}\right)}-I\right) A x_{n}\right)-\left(x_{n}+\xi A^{*}\left(T_{r_{n+1}}^{\left(F_{2}, h_{2}\right)}-\right.\right.$ I) $\left.A x_{n}\right) \|$.

Furthermore, one has

$$
\begin{aligned}
\left\|y_{n+1}-y_{n}\right\|= & \left\|u_{n+1}+\alpha d_{n+2}-\left(u_{n}+\alpha d_{n+1}\right)\right\| \\
\leq & \left\|T_{n+1} u_{n+1}-T_{n} u_{n}\right\| \\
& +\alpha\left\|\gamma_{n+1} d_{n+1}-\gamma_{n} d_{n}\right\| \\
\leq & \left\|T_{n+1} u_{n+1}-T_{n+1} u_{n}\right\| \\
& +\left\|T_{n+1} u_{n}-T_{n} u_{n}\right\| \\
& +\alpha\left\|\gamma_{n+1} d_{n+1}-\gamma_{n} d_{n}\right\| \\
\leq & \left\|u_{n+1}-u_{n}\right\|+\left\|T_{n+1} u_{n}-T_{n} u_{n}\right\| \\
& +\alpha M_{1}\left(\gamma_{n+1}+\gamma_{n}\right)
\end{aligned}
$$


It follows from (34) that

$$
\begin{aligned}
\left\|T_{n+1} u_{n}-T_{n} u_{n}\right\|=\| \frac{P_{C}\left(I-\lambda_{n+1} B\right)-\left(1-b_{n+1}\right) I}{b_{n+1}} u_{n} \\
-\frac{P_{C}\left(I-\lambda_{n} B\right)-\left(1-b_{n}\right) I}{b_{n}} u_{n} \| \\
=\| \frac{4 \beta P_{C}\left(I-\lambda_{n+1} B\right)-\left(2 \beta-\lambda_{n+1}\right) I}{2 \beta+\lambda_{n+1}} u_{n} \\
-\frac{4 \beta P_{C}\left(I-\lambda_{n} B\right)-\left(2 \beta-\lambda_{n}\right) I}{2 \beta+\lambda_{n}} u_{n}\|=\| \frac{4 \beta P_{C}\left(I-\lambda_{n+1} B\right)}{2 \beta+\lambda_{n+1}} u_{n} \\
-\frac{4 \beta P_{C}\left(I-\lambda_{n} B\right)}{2 \beta+\lambda_{n}} u_{n}+\frac{\left(2 \beta-\lambda_{n}\right)}{2 \beta+\lambda_{n}} u_{n}-\frac{\left(2 \beta-\lambda_{n+1}\right)}{2 \beta+\lambda_{n+1}} u_{n} \| \\
\leq \| \frac{4 \beta\left[\left(2 \beta+\lambda_{n}\right) P_{C}\left(I-\lambda_{n+1} B\right)-\left(2 \beta+\lambda_{n+1}\right) P_{C}\left(I-\lambda_{n} B\right)\right]}{\left(2 \beta+\lambda_{n+1}\right)\left(2 \beta+\lambda_{n}\right)} \\
\cdot u_{n}\|+\| \frac{4 \beta\left(\lambda_{n+1}-\lambda_{n}\right)}{\left(2 \beta+\lambda_{n+1}\right)\left(2 \beta+\lambda_{n}\right)} u_{n} \| \\
\quad \leq\left\|\frac{4 \beta\left(\lambda_{n}-\lambda_{n+1}\right) P_{C}\left(I-\lambda_{n+1} B\right)}{\left(2 \beta+\lambda_{n+1}\right)\left(2 \beta+\lambda_{n}\right)} u_{n}\right\| \\
+\left\|\frac{4 \beta\left(2 \beta+\lambda_{n+1}\right)\left[P_{C}\left(I-\lambda_{n+1} B\right)-P_{C}\left(I-\lambda_{n} B\right)\right]}{\left(2 \beta+\lambda_{n+1}\right)\left(2 \beta+\lambda_{n}\right)} u_{n}\right\| \\
+\left\|\frac{4 \beta\left(\lambda_{n+1}-\lambda_{n}\right)}{\left(2 \beta+\lambda_{n+1}\right)\left(2 \beta+\lambda_{n}\right)} u_{n}\right\| \leq \frac{1}{\beta}\left|\lambda_{n}-\lambda_{n+1}\right| \\
+\left\|P_{C}\left(I-\lambda_{n+1} B\right) u_{n}\right\|+4 \beta\left\|B u_{n}\right\| \\
\left.+u_{n} \|\right) \leq M_{2}\left|\lambda_{n+1}-\lambda_{n}\right|,
\end{aligned}
$$

where $M_{2}=\sup _{n \in N}\left\{(1 / \beta)\left(\left\|P_{C}\left(I-\lambda_{n+1} B\right) u_{n}\right\|+4 \beta\left\|B u_{n}\right\|+\right.\right.$ $\left.\left.\left\|u_{n}\right\|\right)\right\}$.

Hence from (54)-(56), we get

$$
\begin{aligned}
\left\|y_{n+1}-y_{n}\right\| \leq & \left\|x_{n+1}-x_{n}\right\|+\xi\|A\| \sigma_{n}+\zeta_{n} \\
& +M_{2}\left|\lambda_{n+1}-\lambda_{n}\right|+\alpha M_{1}\left(\gamma_{n+1}+\gamma_{n}\right) .
\end{aligned}
$$

Set $x_{n+1}=\left(1-\beta_{n}\right) z_{n}+\beta_{n} x_{n}$; it follows that

$$
\begin{aligned}
z_{n} & =\frac{x_{n+1}-\beta_{n} x_{n}}{1-\beta_{n}} \\
& =\frac{\alpha_{n} \gamma f\left(x_{n}\right)+\left(\left(1-\beta_{n}\right) I-\alpha_{n} D\right) y_{n}}{1-\beta_{n}} .
\end{aligned}
$$

As a result,

$$
\begin{aligned}
& \left\|z_{n+1}-z_{n}\right\| \\
& =\| \frac{\alpha_{n+1} \gamma f\left(x_{n+1}\right)+\left(\left(1-\beta_{n+1}\right) I-\alpha_{n+1} D\right) y_{n+1}}{1-\beta_{n+1}} \\
& -\frac{\alpha_{n} \gamma f\left(x_{n}\right)+\left(\left(1-\beta_{n}\right) I-\alpha_{n} D\right) y_{n}}{1-\beta_{n}} \|
\end{aligned}
$$

$$
\begin{aligned}
& =\| \frac{\alpha_{n+1}\left(\gamma f\left(x_{n+1}\right)-D y_{n+1}\right)}{1-\beta_{n+1}} \\
& -\frac{\alpha_{n}\left(\gamma f\left(x_{n}\right)-D y_{n}\right)}{1-\beta_{n}}+y_{n+1}-y_{n} \| \\
& \leq \frac{\alpha_{n+1}}{1-\beta_{n+1}}\left\|\gamma f\left(x_{n+1}\right)-D y_{n+1}\right\| \\
& +\frac{\alpha_{n}}{1-\beta_{n}}\left\|\gamma f\left(x_{n}\right)-D y_{n}\right\|+\left\|y_{n+1}-y_{n}\right\| \\
& \leq \frac{\alpha_{n+1}}{1-\beta_{n+1}}\left\|\gamma f\left(x_{n+1}\right)-D y_{n+1}\right\| \\
& +\frac{\alpha_{n}}{1-\beta_{n}}\left\|\gamma f\left(x_{n}\right)-D y_{n}\right\|+\left\|x_{n+1}-x_{n}\right\|+\xi\|A\| \\
& \cdot \sigma_{n}+\zeta_{n}+M_{2}\left|\lambda_{n+1}-\lambda_{n}\right|+\alpha M_{1}\left(\gamma_{n+1}+\gamma_{n}\right) .
\end{aligned}
$$

Letting $n \longrightarrow \infty$, from (C1)-(C5), we have

$$
\limsup _{n \rightarrow \infty}\left(\left\|z_{n+1}-z_{n}\right\|-\left\|x_{n+1}-x_{n}\right\|\right) \leq 0 .
$$

By Lemma 12, we obtain

$$
\lim _{n \rightarrow \infty}\left\|z_{n}-x_{n}\right\|=0 .
$$

Further,

$$
\lim _{n \longrightarrow \infty}\left\|x_{n+1}-x_{n}\right\|=\lim _{n \longrightarrow \infty}\left(1-\beta_{n}\right)\left\|z_{n}-x_{n}\right\|=0 .
$$

Step 3 (we show that $\lim _{n \rightarrow \infty}\left\|u_{n}-x_{n}\right\|=0$ ). Since $x^{*} \in \Omega$, $x^{*}=T_{r_{n}}^{\left(F_{1}, h_{1}\right)} x^{*}$, and $T_{r_{n}}^{\left(F_{1}, h_{1}\right)}$ is firmly nonexpansive, we obtain

$$
\begin{aligned}
& \left\|u_{n}-x^{*}\right\|^{2}=\| T_{r_{n}}^{\left(F_{1}, h_{1}\right)}\left(x_{n}+\xi A^{*}\left(T_{r_{n}}^{\left(F_{2}, h_{2}\right)}-I\right) A x_{n}\right) \\
& -x^{*}\left\|^{2}=\right\| T_{r_{n}}^{\left(F_{1}, h_{1}\right)}\left(x_{n}+\xi A^{*}\left(T_{r_{n}}^{\left(F_{2}, h_{2}\right)}-I\right) A x_{n}\right) \\
& -T_{r_{n}}^{\left(F_{1}, h_{1}\right)} x^{*} \|^{2} \leq\left\langle u_{n}-x^{*}, x_{n}+\xi A^{*}\left(T_{r_{n}}^{\left(F_{2}, h_{2}\right)}-I\right)\right. \\
& \left.\cdot A x_{n}-x^{*}\right\rangle=\frac{1}{2}\left\{\left\|u_{n}-x^{*}\right\|^{2}+\| x_{n}\right. \\
& +\xi A^{*}\left(T_{r_{n}}^{\left(F_{2}, h_{2}\right)}-I\right) A x_{n}-x^{*}\left\|^{2}-\right\|\left(u_{n}-x^{*}\right) \\
& \left.-\left[x_{n}+\xi A^{*}\left(T_{r_{n}}^{\left(F_{2}, h_{2}\right)}-I\right) A x_{n}-x^{*}\right] \|^{2}\right\}=\frac{1}{2}\left\{\| u_{n}\right. \\
& -x^{*}\left\|^{2}+\right\| x_{n}-x^{*}\left\|^{2}-\right\| u_{n}-x_{n} \|^{2}+2 \xi\left\langle u_{n}\right. \\
& \left.\left.-x^{*}, A^{*}\left(T_{r_{n}}^{\left(F_{2}, h_{2}\right)}-I\right) A x_{n}\right\rangle\right\} .
\end{aligned}
$$

Hence, we obtain

$$
\begin{aligned}
& \left\|u_{n}-x^{*}\right\|^{2} \\
& \leq\left\|x_{n}-x^{*}\right\|^{2}-\left\|u_{n}-x_{n}\right\|^{2} \\
& \quad+2 \xi\left\|A\left(u_{n}-x^{*}\right)\right\|\left\|\left(T_{r_{n}}^{\left(F_{2}, h_{2}\right)}-I\right) A x_{n}\right\| .
\end{aligned}
$$


Furthermore,

$$
\begin{aligned}
\left\|y_{n}-x^{*}\right\|^{2} & =\left\|u_{n}+\alpha d_{n+1}-x^{*}\right\|^{2} \\
& \leq\left\|u_{n}-x^{*}\right\|^{2}+2 \alpha \gamma_{n}\left\langle y_{n}-x^{*}, d_{n}\right\rangle \\
& \leq\left\|u_{n}-x^{*}\right\|^{2}+M_{3} \gamma_{n},
\end{aligned}
$$

where $M_{3}=\sup _{n \in N} 2 \alpha\left\langle y_{n}-x^{*}, d_{n}\right\rangle$.

By Lemma 1 (iii), (46), and (65), we have

$$
\begin{aligned}
& \left\|x_{n+1}-x^{*}\right\|^{2}=\| \alpha_{n} \gamma f\left(x_{n}\right)+\beta_{n} x_{n} \\
& +\left(\left(1-\beta_{n}\right) I-\alpha_{n} D\right) y_{n}-x^{*} \|^{2} \\
& =\| \alpha_{n}\left(\gamma f\left(x_{n}\right)-D x^{*}\right)+\beta_{n}\left(x_{n}-x^{*}\right) \\
& +\left(\left(1-\beta_{n}\right) I-\alpha_{n} D\right)\left(y_{n}-x^{*}\right) \|^{2} \\
& \quad \leq\left(\alpha_{n}\left\|\gamma f\left(x_{n}\right)-D x^{*}\right\|+\beta_{n}\left\|x_{n}-x^{*}\right\|\right. \\
& \left.+\left(1-\beta_{n}-\alpha_{n} \bar{\gamma}\right)\left\|y_{n}-x^{*}\right\|\right)^{2} \\
& =\left(\alpha_{n} \bar{\gamma}\left\|\frac{1}{\bar{\gamma}}\left(\gamma f\left(x_{n}\right)-D x^{*}\right)\right\|+\beta_{n}\left\|x_{n}-x^{*}\right\|\right. \\
& \left.+\left(1-\beta_{n}-\alpha_{n} \bar{\gamma}\right)\left\|y_{n}-x^{*}\right\|\right)^{2} \leq \alpha_{n} \bar{\gamma} \frac{1}{\bar{\gamma}^{2}} \| \gamma f\left(x_{n}\right) \\
& -D x^{*}\left\|^{2}+\beta_{n}\right\| x_{n}-x^{*}\left\|^{2}+\left(1-\beta_{n}-\alpha_{n} \bar{\gamma}\right)\right\| y_{n} \\
& -x^{*}\left\|^{2} \leq \frac{\alpha_{n}}{\bar{\gamma}}\right\| \gamma f\left(x_{n}\right)-D x^{*}\left\|^{2}+\beta_{n}\right\| x_{n}-x^{*} \|^{2} \\
& +\left(1-\beta_{n}-\alpha_{n} \bar{\gamma}\right)\left\|y_{n}-x^{*}\right\|^{2} \leq \frac{\alpha_{n}}{\bar{\gamma}} \| \gamma f\left(x_{n}\right) \\
& +D x^{*}\left\|^{2}+\beta_{n}\right\| x_{n}-x^{*} \|^{2}+\left(1-\beta_{n}-\alpha_{n} \bar{\gamma}\right) \\
& +\left(\left\|x_{n}-x^{*}\right\|^{2}+\xi(L \xi-1)\left\|\left(T_{r_{n}}^{\left(F_{2}, h_{2}\right)}-I\right) A x_{n}\right\|^{2}\right. \\
& \left.+M_{3} \gamma_{n}\right)=\frac{\alpha_{n}}{\bar{\gamma}}\left\|\gamma f\left(x_{n}\right)-D x^{*}\right\|^{2}+\beta_{n}\left\|x_{n}-x^{*}\right\|^{2} \\
& \left.+\left(1-\beta_{n}-\alpha_{n} \bar{\gamma}\right)\left\|x_{n}-x^{*}\right\|_{n}^{2}-\left(1-\beta_{n}\right)-\alpha_{n} \bar{\gamma}\right) \xi(1) A x_{n} \|^{2}+\left(1-\beta_{n}-\alpha_{n} \bar{\gamma}\right) M_{3} \gamma_{n} .
\end{aligned}
$$

As a result,

$$
\begin{aligned}
(1- & \left.\beta_{n}-\alpha_{n} \bar{\gamma}\right) \xi(1-L \xi)\left\|\left(T_{r_{n}}^{\left(F_{2}, h_{2}\right)}-I\right) A x_{n}\right\|^{2} \\
\leq & \left\|x_{n}-x^{*}\right\|^{2}-\left\|x_{n+1}-x^{*}\right\|^{2} \\
& +\frac{\alpha_{n}}{\bar{\gamma}}\left\|\gamma f\left(x_{n}\right)-D x^{*}\right\|^{2}+\left(1-\beta_{n}-\alpha_{n} \bar{\gamma}\right) M_{3} \gamma_{n} \\
\leq & \left(\left\|x_{n}-x^{*}\right\|+\left\|x_{n+1}-x^{*}\right\|\right)\left\|x_{n}-x_{n+1}\right\| \\
& +\frac{\alpha_{n}}{\bar{\gamma}}\left\|\gamma f\left(x_{n}\right)-D x^{*}\right\|^{2}+\left(1-\beta_{n}-\alpha_{n} \bar{\gamma}\right) M_{3} \gamma_{n} .
\end{aligned}
$$

According to $\alpha_{n} \longrightarrow 0, \gamma_{n}=o\left(\alpha_{n}\right),\left(1-\beta_{n}-\alpha_{n} \bar{\gamma}\right) \xi(1-L \xi)>$ 0 , and $\lim _{n \rightarrow \infty}\left\|x_{n+1}-x_{n}\right\|=0$, we obtain

$$
\lim _{n \longrightarrow \infty}\left\|\left(T_{r_{n}}^{\left(F_{2}, h_{2}\right)}-I\right) A x_{n}\right\|=0 .
$$

From (65), (64), and Lemma 1 (iii), we obtain

$$
\begin{aligned}
& \left\|x_{n+1}-x^{*}\right\|^{2}=\| \alpha_{n} \gamma f\left(x_{n}\right)+\beta_{n} x_{n} \\
& +\left(\left(1-\beta_{n}\right) I-\alpha_{n} D\right) y_{n}-x^{*} \|^{2} \\
& =\| \alpha_{n}\left(\gamma f\left(x_{n}\right)-D x^{*}\right)+\beta_{n}\left(x_{n}-x^{*}\right) \\
& +\left(\left(1-\beta_{n}\right) I-\alpha_{n} D\right)\left(y_{n}-x^{*}\right) \|^{2} \\
& \leq\left(\alpha_{n}\left\|\gamma f\left(x_{n}\right)-D x^{*}\right\|+\beta_{n}\left\|x_{n}-x^{*}\right\|\right. \\
& \left.+\left(1-\beta_{n}-\alpha_{n} \bar{\gamma}\right)\left\|y_{n}-x^{*}\right\|\right)^{2} \\
& =\left(\alpha_{n} \bar{\gamma}\left\|\frac{1}{\bar{\gamma}}\left(\gamma f\left(x_{n}\right)-D x^{*}\right)\right\|+\beta_{n}\left\|x_{n}-x^{*}\right\|\right. \\
& \left.+\left(1-\beta_{n}-\alpha_{n} \bar{\gamma}\right)\left\|y_{n}-x^{*}\right\|\right)^{2} \leq \alpha_{n} \bar{\gamma} \frac{1}{\bar{\gamma}^{2}} \| \gamma f\left(x_{n}\right) \\
& -D x^{*}\left\|^{2}+\beta_{n}\right\| x_{n}-x^{*}\left\|^{2}+\left(1-\beta_{n}-\alpha_{n} \bar{\gamma}\right)\right\| y_{n} \\
& -x^{*}\left\|^{2} \leq \frac{\alpha_{n}}{\bar{\gamma}}\right\| \gamma f\left(x_{n}\right)-D x^{*}\left\|^{2}+\beta_{n}\right\| x_{n}-x^{*} \|^{2} \\
& +\left(1-\beta_{n}-\alpha_{n} \bar{\gamma}\right)\left\|y_{n}-x^{*}\right\|^{2} \leq \frac{\alpha_{n}}{\bar{\gamma}} \| \gamma f\left(x_{n}\right) \\
& + \\
& +
\end{aligned}
$$

Therefore, one has

$$
\begin{aligned}
& \left(1-\beta_{n}-\alpha_{n} \bar{\gamma}\right)\left\|u_{n}-x_{n}\right\|^{2} \leq \frac{\alpha_{n}}{\bar{\gamma}}\left\|\gamma f\left(x_{n}\right)-D x^{*}\right\|^{2} \\
& \quad+\left(1-\alpha_{n} \bar{\gamma}\right)\left\|x_{n}-x^{*}\right\|^{2}-\left\|x_{n+1}-x^{*}\right\|^{2} \\
& \quad+2 \xi\left(1-\beta_{n}-\alpha_{n} \bar{\gamma}\right)\left\|A\left(u_{n}-x^{*}\right)\right\|
\end{aligned}
$$




$$
\begin{aligned}
& \cdot\left\|\left(T_{r_{n}}^{\left(F_{2}, h_{2}\right)}-I\right) A x_{n}\right\|+\left(1-\beta_{n}-\alpha_{n} \bar{\gamma}\right) M_{3} \gamma_{n} \\
& \leq \frac{\alpha_{n}}{\bar{\gamma}}\left\|\gamma f\left(x_{n}\right)-D x^{*}\right\|^{2}+\left\|x_{n}-x^{*}\right\|^{2} \\
& -\left\|x_{n+1}-x^{*}\right\|^{2}+2 \xi\left(1-\beta_{n}-\alpha_{n} \bar{\gamma}\right)\left\|A\left(u_{n}-x^{*}\right)\right\| \\
& \cdot\left\|\left(T_{r_{n}}^{\left(F_{2}, h_{2}\right)}-I\right) A x_{n}\right\|+\left(1-\beta_{n}-\alpha_{n} \bar{\gamma}\right) M_{3} \gamma_{n} \\
& \leq \frac{\alpha_{n}}{\bar{\gamma}}\left\|\gamma f\left(x_{n}\right)-D x^{*}\right\|^{2}+\left\|x_{n}-x_{n+1}\right\| \\
& \cdot\left(\left\|x_{n}-x^{*}\right\|+\left\|x_{n+1}-x^{*}\right\|\right)+2 \xi\left(1-\beta_{n}-\alpha_{n} \bar{\gamma}\right) \\
& \cdot\left\|A\left(u_{n}-x^{*}\right)\right\|\left\|\left(T_{r_{n}}^{\left(F_{2}, h_{2}\right)}-I\right) A x_{n}\right\| \\
& +\left(1-\beta_{n}-\alpha_{n} \bar{\gamma}\right) M_{3} \gamma_{n} .
\end{aligned}
$$

According to $\alpha_{n} \longrightarrow 0, \gamma_{n}=o\left(\alpha_{n}\right),(62)$, and (68), we obtain

$$
\lim _{n \rightarrow \infty}\left\|u_{n}-x_{n}\right\|=0 \text {. }
$$

Step 4 (we show that $\lim _{n \rightarrow \infty}\left\|T_{n} u_{n}-u_{n}\right\|=0$ ). From (58), we have

$$
z_{n}-y_{n}=\frac{\alpha_{n}}{1-\beta_{n}} \gamma f\left(x_{n}\right)-\frac{\alpha_{n}}{1-\beta_{n}} D y_{n}
$$

Hence,

$$
\left\|z_{n}-y_{n}\right\| \leq \frac{\alpha_{n} \gamma}{1-\beta_{n}}\left|f\left(x_{n}\right)\right|+\frac{\alpha_{n}}{1-\beta_{n}}\left\|D y_{n}\right\| .
$$

From (C1) and (C2), one has

$$
\lim _{n \longrightarrow \infty}\left\|z_{n}-y_{n}\right\|=0 \text {. }
$$

By (74) and (61), we have

$$
\lim _{n \longrightarrow \infty}\left\|x_{n}-y_{n}\right\|=0 .
$$

Combining (71) and (75), one has

$$
\lim _{n \longrightarrow \infty}\left\|u_{n}-y_{n}\right\|=0 .
$$

Noting that

$$
y_{n}-u_{n}=T_{n} u_{n}-u_{n}+\alpha \gamma_{n} d_{n}
$$

one has

$$
T_{n} u_{n}-u_{n}=y_{n}-u_{n}-\alpha \gamma_{n} d_{n} .
$$

It follows from (76) and $\alpha_{n} \longrightarrow 0, \gamma_{n}=o\left(\alpha_{n}\right)$ that

$$
\lim _{n \rightarrow \infty}\left\|T_{n} u_{n}-u_{n}\right\|=0
$$

We claim that

$$
\limsup _{n \rightarrow \infty}\left\langle(D-\gamma f) q, q-x_{n}\right\rangle \leq 0,
$$

where $q$ is the unique solution of the variational inequality $\langle(D-\gamma f) q, x-q\rangle \geq 0, \forall x \in \Omega$.

To show this inequality, we choose a subsequence $\left\{x_{n_{i}}\right\}$ of $\left\{x_{n}\right\}$ such that

$$
\begin{aligned}
& \limsup _{n \rightarrow \infty}\left\langle(D-\gamma f) q, q-x_{n}\right\rangle \\
& \quad=\lim _{i \rightarrow \infty}\left\langle(D-\gamma f) q, q-x_{n_{i}}\right\rangle .
\end{aligned}
$$

Since $\left\{x_{n_{i}}\right\}$ is bounded, there exists a subsequence of $\left\{x_{n_{i}}\right\}$ which converges weakly to $z \in C$. Without loss of generality, we can assume that $x_{n_{i}} \rightarrow z$.

Step 5 (we show that $z \in \Omega$ ). First, we show that $z \in \operatorname{VI}(C, B)$. Let $M: H_{1} \longrightarrow 2^{H_{1}}$ be a set-valued mapping defined by

$$
M v= \begin{cases}B v+N_{C} v, & v \in C, \\ \emptyset, & v \notin C,\end{cases}
$$

where $N_{C} v:=\left\{z \in H_{1}:\langle v-u, z\rangle \geq 0, \forall u \in C\right\}$ is the normal cone to $C$ at $v \in C$. Then $M$ is maximal monotone and $0 \in$ $M v$ if and only if $v \in \operatorname{VI}(C, B)$ (see [36]). Let $(v, u) \in G(M)$. Therefore, we have

$$
u \in M v=B v+N_{C} v,
$$

and so

$$
u-B v \in N_{C} v
$$

According to $u_{n} \in C$, we obtain

$$
\left\langle v-u_{n}, u-B v\right\rangle \geq 0 \text {. }
$$

On the other hand, according to

$$
P_{C}\left(\left(I-\lambda_{n} B\right) u_{n}\right)=u_{n}+b_{n}\left(T_{n} u_{n}-u_{n}\right)
$$

where $b_{n}=\left(2 \beta+\lambda_{n}\right) / 4 \beta$, for $\forall n \in N$, and $v \in H_{1}$, we have

$$
\begin{aligned}
& \left\langle v-u_{n}-b_{n}\left(T_{n} u_{n}-u_{n}\right), u_{n}+b_{n}\left(T_{n} u_{n}-u_{n}\right)\right. \\
& \left.-\left(u_{n}-\lambda_{n} B u_{n}\right)\right\rangle \geq 0 .
\end{aligned}
$$

Therefore,

$$
\begin{gathered}
\left\langle v-u_{n}, b_{n}\left(T_{n} u_{n}-u_{n}\right)+\lambda_{n} B u_{n}\right\rangle-\left\|b_{n}\left(T_{n} u_{n}-u_{n}\right)\right\|^{2} \\
-\left\langle b_{n}\left(T_{n} u_{n}-u_{n}\right), \lambda_{n} B u_{n}\right\rangle \geq 0 .
\end{gathered}
$$

As a result,

$$
\begin{array}{r}
\left\langle v-u_{n}, \frac{b_{n}}{\lambda_{n}}\left(T_{n} u_{n}-u_{n}\right)+B u_{n}\right\rangle \\
-\left\langle b_{n}\left(T_{n} u_{n}-u_{n}\right), B u_{n}\right\rangle \geq 0 .
\end{array}
$$



has

Furthermore, according to (85) and (89), for $\forall n \in N$, one

$$
\begin{aligned}
\left\langle v-u_{n}, u\right\rangle \geq & \left\langle v-u_{n}, B v\right\rangle \\
& -\left\langle v-u_{n}, \frac{b_{n}}{\lambda_{n}}\left(T_{n} u_{n}-u_{n}\right)+B u_{n}\right\rangle \\
& +\left\langle b_{n}\left(T_{n} u_{n}-u_{n}\right), B u_{n}\right\rangle \\
= & \left\langle v-u_{n}, B v-B u_{n}\right\rangle \\
& -\left\langle v-u_{n}, \frac{b_{n}}{\lambda_{n}}\left(T_{n} u_{n}-u_{n}\right)\right\rangle \\
& +\left\langle b_{n}\left(T_{n} u_{n}-u_{n}\right), B u_{n}\right\rangle .
\end{aligned}
$$

Replacing $n$ by $n_{i}$, one has

$$
\begin{aligned}
\left\langle v-u_{n_{i}}, u\right\rangle \geq & \left\langle v-u_{n_{i}}, B v-B u_{n_{i}}\right\rangle \\
& -\left\langle v-u_{n_{i}}, \frac{b_{n_{i}}}{\lambda_{n_{i}}}\left(T_{n_{i}} u_{n_{i}}-u_{n_{i}}\right)\right\rangle \\
& +\left\langle b_{n_{i}}\left(T_{n_{i}} u_{n_{i}}-u_{n_{i}}\right), B u_{n_{i}}\right\rangle .
\end{aligned}
$$

Since $\left\|u_{n}-x_{n}\right\| \longrightarrow 0$ and $x_{n_{i}} \rightarrow z$, we have $u_{n_{i}} \rightarrow z$. Noting that $B$ is $\beta$-ism, from (91) and (79), we have

$$
\langle v-z, u\rangle \geq 0 .
$$

Since $M$ is maximal monotone, one has $z \in M^{-1} 0$. Hence $z \in V I(C, B)$.

Next, we prove $z \in \Gamma$.

According to Algorithm 13, we have

$$
u_{n_{i}}=T_{r_{n_{i}}}^{\left(F_{1}, h_{1}\right)}\left(x_{n_{i}}+\xi A^{*}\left(T_{r_{n_{i}}}^{\left(F_{2}, h_{2}\right)}-I\right) A x_{n_{i}}\right) .
$$

By (8), for any $w \in C$, one has

$$
\begin{aligned}
0 & \leq F_{1}\left(u_{n_{i}}, w\right)+h_{1}\left(u_{n_{i}}, w\right)+\frac{1}{r_{n_{i}}}\left\langle w-u_{n_{i}}, u_{n_{i}}\right. \\
& \left.-\left(x_{n_{i}}+\xi A^{*}\left(T_{r_{n_{i}}}^{\left(F_{2}, h_{2}\right)}-I\right) A x_{n_{i}}\right)\right\rangle=F_{1}\left(u_{n_{i}}, w\right) \\
& +h_{1}\left(u_{n_{i}}, w\right)+\frac{1}{r_{n_{i}}}\left\langle w-u_{n_{i}}, u_{n_{i}}-x_{n_{i}}\right\rangle-\frac{\xi}{r_{n_{i}}}\langle A w \\
& \left.-A u_{n_{i}},\left(T_{r_{n_{i}}}^{\left(F_{2}, h_{2}\right)}-I\right) A x_{n_{i}}\right\rangle \leq F_{1}\left(u_{n_{i}}, w\right) \\
& +h_{1}\left(u_{n_{i}}, w\right)+\frac{1}{r_{n_{i}}}\left\|w-u_{n_{i}}\right\|\left\|u_{n_{i}}-x_{n_{i}}\right\|+\frac{\xi}{r_{n_{i}}} \| A w \\
& -A u_{n_{i}}\|\|\left(T_{r_{n_{i}}}^{\left(F_{2}, h_{2}\right)}-I\right) A x_{n_{i}} \|
\end{aligned}
$$

According to the monotonicity of $F_{1}$, we have

$$
\begin{aligned}
F_{1}\left(w, u_{n_{i}}\right) & \\
\leq & h_{1}\left(u_{n_{i}}, w\right)+\frac{1}{r_{n_{i}}}\left\|w-u_{n_{i}}\right\|\left\|u_{n_{i}}-x_{n_{i}}\right\| \\
& +\frac{\xi}{r_{n_{i}}}\left\|A w-A u_{n_{i}}\right\|\left\|\left(T_{r_{n_{i}}}^{\left(F_{2}, h_{2}\right)}-I\right) A x_{n_{i}}\right\|
\end{aligned}
$$

From Assumption 2 (iv) on $F$ and (ii) on $h$, (68), and (71), one has

$$
F_{1}(w, z) \leq h_{1}(z, w) .
$$

It follows from the monotonicity of $h_{1}$ that

$$
F_{1}(w, z)+h_{1}(w, z) \leq 0, \quad \forall w \in C .
$$

For any $t \in(0,1]$ and $w \in C$, let $w_{t}=t w+(1-t) z$. Since $z \in C$ and $C$ is convex, we obtain that $w_{t} \in C$. Hence

$$
F_{1}\left(w_{t}, z\right)+h_{1}\left(w_{t}, z\right) \leq 0 .
$$

From Assumption $2(i),(i v)$ on $F$ and (i), (iii) on $h$, we have

$$
\begin{aligned}
0 \leq & F_{1}\left(w_{t}, w_{t}\right)+h_{1}\left(w_{t}, w_{t}\right) \\
\leq & t\left[F_{1}\left(w_{t}, w\right)+h_{1}\left(w_{t}, w\right)\right] \\
& +(1-t)\left[F_{1}\left(w_{t}, z\right)+h_{1}\left(w_{t}, z\right)\right] \\
\leq & t\left[F_{1}\left(w_{t}, w\right)+h_{1}\left(w_{t}, w\right)\right],
\end{aligned}
$$

which implies that

$$
F_{1}\left(w_{t}, w\right)+h_{1}\left(w_{t}, w\right) \geq 0, \quad \forall w \in C .
$$

Letting $t \longrightarrow 0$ and by Assumption 2 (iii) on $F$ and (ii) on $h$, we obtain

$$
F_{1}(z, w)+h_{1}(z, w) \geq 0, \quad \forall w \in C ;
$$

that is, $z \in \operatorname{GEP}\left(F_{1}, h_{1}\right)$.

As follows, we prove $A z \in \operatorname{GEP}\left(F_{2}, h_{2}\right)$.

Since $A$ is a bounded linear operator, one has $A x_{n_{i}} \rightarrow A z$.

Now, set $\zeta_{n_{i}}=A x_{n_{i}}-T_{r_{n_{i}}}^{\left(F_{2}, h_{2}\right)} A x_{n_{i}}$. It follows from (68) that $\lim _{i \rightarrow \infty} \zeta_{n_{i}}=0$. Since $A x_{n_{i}}-\zeta_{n_{i}}=T_{r_{n_{i}}}^{\left(F_{2}, h_{2}\right)} A x_{n_{i}}$, by (9) we have

$$
\begin{aligned}
F_{2}\left(A x_{n_{i}}-\zeta_{n_{i}}, \widetilde{z}\right)+h_{2}\left(A x_{n_{i}}-\zeta_{n_{i}}, \widetilde{z}\right) & \\
& +\frac{1}{r_{n_{i}}}\left\langle\widetilde{z}-\left(A x_{n_{i}}-\zeta_{n_{i}}\right),\left(A x_{n_{i}}-\zeta_{n_{i}}\right)-A x_{n_{i}}\right\rangle
\end{aligned}
$$

$\geq 0, \quad \forall \widetilde{z} \in Q$.

Furthermore, one has

$$
\begin{aligned}
F_{2}\left(A x_{n_{i}}-\zeta_{n_{i}}, \widetilde{z}\right)+h_{2}\left(A x_{n_{i}}-\zeta_{n_{i}}, \tilde{z}\right) \\
\quad+\frac{1}{r_{n_{i}}}\left\langle\widetilde{z}-A x_{n_{i}}+\zeta_{n_{i}},-\zeta_{n_{i}}\right\rangle \geq 0, \quad \forall \widetilde{z} \in Q .
\end{aligned}
$$


From the upper semicontinuity of $F_{2}(x, y)$ and $h_{2}(x, y)$ on $x$, we have

$$
F_{2}(A z, \widetilde{z})+h_{2}(A z, \widetilde{z}) \geq 0, \quad \forall \widetilde{z} \in Q,
$$

which means that $A z \in \operatorname{GEP}\left(F_{2}, h_{2}\right)$. As a result, $z \in \Gamma$.

Therefore, $z \in \Omega$.

Since $q=P_{\Omega}(I-D+\gamma f) q$ is the unique solution of the variational inequality problem $\langle(D-\gamma f) q, x-q\rangle \geq 0, \forall x \in \Omega$, by (81) and $z \in \Omega$, we have

$$
\limsup _{n \rightarrow \infty}\left\langle(D-\gamma f) q, q-x_{n}\right\rangle \leq 0 .
$$

Step 6 (finally, we show that $\left\{x_{n}\right\}$ converges strongly to q). It is obvious that

$$
\begin{aligned}
\left\|y_{n}-q\right\| & =\left\|T_{n} u_{n}-q+\alpha \gamma_{n} d_{n}\right\| \\
& \leq\left\|T_{n} u_{n}-T_{n} q\right\|+\alpha \gamma_{n}\left\|d_{n}\right\| \\
& \leq\left\|u_{n}-q\right\|+\alpha M_{1} \gamma_{n}=\left\|u_{n}-q\right\|+M_{4} \gamma_{n} \\
& \leq\left\|x_{n}-q\right\|+M_{4} \gamma_{n},
\end{aligned}
$$

where $M_{4}=\alpha M_{1}$. And the first inequality is true because $q \epsilon$ $\Omega$ and $T_{n} q=q$ according to the same reasoning to equality (50). The last inequality is obtained by $q \in \Omega$ and the same reasoning to inequality (47). Thus, from Lemma $1(i)$, we have

$$
\begin{aligned}
& \left\|x_{n+1}-q\right\|^{2}=\| \alpha_{n} \gamma f\left(x_{n}\right)+\beta_{n} x_{n} \\
& \quad+\left(\left(1-\beta_{n}\right) I-\alpha_{n} D\right) y_{n}-q \|^{2} \\
& \quad=\| \alpha_{n}\left(\gamma f\left(x_{n}\right)-D q\right)+\beta_{n}\left(x_{n}-q\right) \\
& \quad+\left(\left(1-\beta_{n}\right) I-\alpha_{n} D\right)\left(y_{n}-q\right)\left\|^{2} \leq\right\| \beta_{n}\left(x_{n}-q\right) \\
& \quad+\left(\left(1-\beta_{n}\right) I-\alpha_{n} D\right)\left(y_{n}-q\right) \|^{2}+2 \alpha_{n}\left\langle\gamma f\left(x_{n}\right)\right. \\
& \left.\quad-D q, x_{n+1}-q\right\rangle \leq\left(\beta_{n}\left\|x_{n}-q\right\|\right. \\
& \left.\left.\quad+\left(1-\beta_{n}-\alpha_{n} \bar{\gamma}\right) \| y_{n}-q\right) \|\right)^{2}+2 \alpha_{n} \gamma\left\langle f\left(x_{n}\right)\right. \\
& \left.\quad-f(q), x_{n+1}-q\right\rangle+2 \alpha_{n}\left\langle\gamma f(q)-D q, x_{n+1}-q\right\rangle \\
& \quad \leq\left(\left(1-\alpha_{n} \bar{\gamma}\right)\left\|x_{n}-q\right\|+\left(1-\beta_{n}-\alpha_{n} \bar{\gamma}\right) M_{4} \gamma_{n}\right)^{2} \\
& \quad+2 \alpha_{n} \gamma \eta\left\|x_{n}-q\right\|\left\|x_{n+1}-q\right\|+2 \alpha_{n}\langle\gamma f(q) \\
& \left.\quad-D q, x_{n+1}-q\right\rangle \leq\left(1-\alpha_{n} \bar{\gamma}\right)^{2}\left\|x_{n}-q\right\|^{2}+M_{5} \gamma_{n} \\
& \quad+\alpha_{n} \gamma \eta\left\|x_{n}-q\right\|^{2}+\alpha_{n} \gamma \eta\left\|x_{n+1}-q\right\|^{2} \\
& \quad+2 \alpha_{n}\left\langle\gamma f(q)-D q, x_{n+1}-q\right\rangle,
\end{aligned}
$$

where $M_{5}=\sup _{n \in N}\left\{2\left(1-\alpha_{n} \bar{\gamma}\right)\left(1-\beta_{n}-\alpha_{n} \bar{\gamma}\right) M_{4}\left\|x_{n}-q\right\|+\right.$ $\left.\left(1-\beta_{n}-\alpha_{n} \bar{\gamma}\right)^{2} M_{4}^{2} \gamma_{n}\right\}$.

As a result,

$$
\begin{aligned}
& \left(1-\alpha_{n} \gamma \eta\right)\left\|x_{n+1}-q\right\|^{2} \\
& \quad \leq\left(1-2 \alpha_{n} \bar{\gamma}+\alpha_{n}^{2} \bar{\gamma}^{2}+\alpha_{n} \gamma \eta\right)\left\|x_{n}-q\right\|^{2}+M_{5} \gamma_{n} \\
& \quad+2 \alpha_{n}\left\langle\gamma f(q)-D q, x_{n+1}-q\right\rangle,
\end{aligned}
$$

which implies that

$$
\begin{aligned}
& \left\|x_{n+1}-q\right\|^{2} \leq\left(1-\frac{2(\bar{\gamma}-\gamma \eta) \alpha_{n}}{1-\alpha_{n} \gamma \eta}\right)\left\|x_{n}-q\right\|^{2} \\
& +\frac{2(\bar{\gamma}-\gamma \eta) \alpha_{n}}{1-\alpha_{n} \gamma \eta}\left(\frac{\alpha_{n} \bar{\gamma}^{2}\left\|x_{n}-q\right\|^{2}+M_{5}\left(\gamma_{n} / \alpha_{n}\right)}{2(\bar{\gamma}-\gamma \eta)}\right. \\
& \left.+\frac{1}{\bar{\gamma}-\gamma \eta}\left\langle\gamma f(q)-D q, x_{n+1}-q\right\rangle\right)=\left(1-t_{n}\right) \\
& \cdot\left\|x_{n}-q\right\|^{2}+t_{n} \delta_{n},
\end{aligned}
$$

where $t_{n}=2(\bar{\gamma}-\gamma \eta) \alpha_{n} /\left(1-\alpha_{n} \gamma \eta\right), \delta_{n}=\left(\alpha_{n} \bar{\gamma}^{2}\left\|x_{n}-q\right\|^{2}+\right.$ $\left.M_{5}\left(\gamma_{n} / \alpha_{n}\right)\right) / 2(\bar{\gamma}-\gamma \eta)+(1 /(\bar{\gamma}-\gamma \eta))\left\langle\gamma f(q)-D q, x_{n+1}-q\right\rangle$. According to (80), $\left(C_{1}\right),\left(C_{3}\right)$, and $\eta \gamma<\bar{\gamma}$, we have $\sum_{n=0}^{\infty} t_{n}=\infty$ and $\lim \sup _{n \rightarrow \infty} \delta_{n} \leq 0$.

By Lemma $7, x_{n} \longrightarrow q$, which completes the proof.

\section{Consequently Results}

In the above section, we discuss the iterative algorithm and prove the strong convergence theorem for finding a common solution of split generalized equilibrium and variational inequality problems. In this section, we give some corollaries, which can find a common solution of the special issues obtained from split generalized equilibrium and variational inequality problems.

If $h_{1}=h_{2}=0$, then SGEP (5)-(6) reduces to the following split equilibrium problem (SEP).

Let $F_{1}: C \times C \longrightarrow R$ and $F_{2}: Q \times Q \longrightarrow R$ be nonlinear bifunctions and $A: H_{1} \longrightarrow H_{2}$ be a bounded linear operator; then SEP is to find $x^{*} \in C$ such that

$$
F_{1}\left(x^{*}, x\right) \geq 0, \quad \forall x \in C,
$$

and such that

$$
y^{*}=A x^{*} \in Q \text { solves } F_{2}\left(y^{*}, y\right) \geq 0, \quad \forall y \in Q .
$$

The solution set of SEP (110)-(111) is denoted by $\Gamma_{1}$. And

$$
\begin{aligned}
& T_{r}^{F_{1}}(x)=\left\{z \in C: F_{1}(z, y)+\frac{1}{r}\langle y-z, z-x\rangle\right. \\
&\geq 0, \forall y \in C\}, \\
& T_{s}^{F_{2}}(w)=\left\{d \in Q: F_{2}(d, e)+\frac{1}{s}\langle e-d, d-w\rangle\right. \\
&\geq 0, \forall e \in Q\} .
\end{aligned}
$$

According to Theorem 14, we can obtain the following corollary.

Corollary 15. Let $H_{1}$ and $H_{2}$ be two real Hilbert spaces and $C \subset H_{1}, Q \subset H_{2}$ be nonempty closed convex subsets. Let $F_{1}$ : $C \times C \longrightarrow R$ and $F_{2}: Q \times Q \longrightarrow R$ satisfy Assumption 2 and $F_{2}$ is upper semicontinuous in the first argument. Assume that 
$\Omega:=\operatorname{VI}(C, B) \cap \Gamma_{1} \neq \emptyset, x_{0} \in H_{1}$ and $\left\{u_{n}\right\},\left\{y_{n}\right\}$, and $\left\{x_{n}\right\}$ are generated by the following iterative scheme:

$$
\begin{aligned}
u_{n} & =T_{r_{n}}^{F_{1}}\left(x_{n}+\xi A^{*}\left(T_{r_{n}}^{F_{2}}-I\right) A x_{n}\right), \\
y_{n} & =u_{n}+\alpha d_{n+1}, \\
x_{n+1} & =\alpha_{n} \gamma f\left(x_{n}\right)+\beta_{n} x_{n}+\left(\left(1-\beta_{n}\right) I-\alpha_{n} D\right) y_{n},
\end{aligned}
$$

where $d_{n+1}=(1 / \alpha)\left(T_{n} u_{n}-u_{n}\right)+\gamma_{n} d_{n}, d_{0}=(1 / \alpha)\left(T_{0} u_{0}-u_{0}\right)$, $\alpha>0$, and $T_{n}$ is defined by (34). Suppose that $\left\{\alpha_{n}\right\},\left\{\beta_{n}\right\} \subset$ $(0,1),\left\{\gamma_{n}\right\} \subset(0,1 / 2),\left\{\lambda_{n}\right\} \subset(0,2 \beta),\left\{r_{n}\right\} \subset(0, \infty)$ satisfy conditions (C1)-(C6) in Theorem 14. Then sequence $\left\{x_{n}\right\}$ converges strongly to $q \in \Omega$, where $q=P_{\Omega}(I-D+\gamma f) q$, which is the unique solution of the variational inequality problem

$$
\langle(D-\gamma f) q, x-q\rangle \geq 0, \quad \forall x \in \Omega,
$$

or equivalently, $q$ is the unique solution to the minimization problem

$$
\min _{x \in Q} \frac{1}{2}\langle D x, x\rangle-h(x)
$$

where $h$ is a potential function for $\gamma f$ such that $h^{\prime}(x)=\gamma f(x)$ for $x \in H_{1}$.

Furthermore, if $F_{1}=F_{2}=F, H_{1}=H_{2}=H$, and $A=$ 0 , then SEP (110)-(111) reduce to the following equilibrium problem (EP).

Let $F: C \times C \longrightarrow R$ be nonlinear bifunction; then EP is to find $x^{*} \in C$ such that

$$
F\left(x^{*}, x\right) \geq 0, \quad \forall x \in C .
$$

The solution set of EP (117) is denoted by $\Gamma_{2}$. And

$$
\begin{aligned}
& T_{r}^{F}(x)=\left\{z \in C: F(z, y)+\frac{1}{r}\langle y-z, z-x\rangle\right. \\
& \quad \geq 0, \forall y \in C\} .
\end{aligned}
$$

According to Corollary 15, let $D=I$, and we can obtain the following corollary.

Corollary 16. Let $H$ be real Hilbert space and $C \subset H$ be nonempty closed convex subset. Let $F: C \times C \longrightarrow R$ satisfy Assumption 2. Assume that $\Omega:=\operatorname{VI}(C, B) \cap \Gamma_{2} \neq \emptyset, x_{0} \in H_{1}$, and $\left\{u_{n}\right\},\left\{y_{n}\right\}$, and $\left\{x_{n}\right\}$ are generated by the following iterative scheme:

$$
\begin{aligned}
u_{n} & =T_{r_{n}}^{F}\left(x_{n}\right), \\
y_{n} & =u_{n}+\alpha d_{n+1}, \\
x_{n+1} & =\alpha_{n} \gamma f\left(x_{n}\right)+\beta_{n} x_{n}+\left(1-\beta_{n}-\alpha_{n}\right) y_{n},
\end{aligned}
$$

where $d_{n+1}=(1 / \alpha)\left(T_{n} u_{n}-u_{n}\right)+\gamma_{n} d_{n}, d_{0}=(1 / \alpha)\left(T_{0} u_{0}-u_{0}\right)$, $\alpha>0$, and $T_{n}$ is defined by (34). Suppose that $\left\{\alpha_{n}\right\},\left\{\beta_{n}\right\} \subset$ $(0,1),\left\{\gamma_{n}\right\} \subset(0,1 / 2),\left\{\lambda_{n}\right\} \subset(0,2 \beta),\left\{r_{n}\right\} \subset(0, \infty)$ satisfy conditions (C1)-(C6) in Theorem 14 . Then sequence $\left\{x_{n}\right\}$ converges strongly to $q \in \Omega$, where $q=P_{\Omega} \gamma f(q)$.
TABLE 1: The final value and the cpu time for different initial points.

\begin{tabular}{lcc}
\hline Init. & \multicolumn{1}{c}{ Fina. } & Sec. \\
\hline$x=(0.7060,0.0318)^{T}$ & $\bar{x}=(4.9924,3.9939)^{T}$ & 1.14 \\
$x=(5.4722,1.3862)^{T}$ & $\bar{x}=(4.9924,3.9939)^{T}$ & 1.35 \\
$x=(89.0903,95.9291)^{T}$ & $\bar{x}=(4.9924,3.9939)^{T}$ & 1.36 \\
\hline
\end{tabular}

\section{Numerical Examples}

In this section, we show some insight into the behavior of Algorithm 13. The whole codes are written in Matlab 7.0. All the numerical results are carried out on a personal Lenovo Thinkpad computer with Intel(R) Core(TM) i7-6500U CPU 2.50GHz and RAM 8.00GB.

Example 1. In the variational inequality problem (3) as well as the split generalized equilibrium problem (5) and (6), let $H_{1}=$ $H_{2}=R^{2}, C=\left\{\left(x_{1}, x_{2}\right)^{T} \in R^{2} \mid 1 \leq x_{1} \leq 5,0.5 \leq x_{2} \leq 4\right\}$, $Q=\left\{\left(y_{1}, y_{2}\right)^{T} \in R^{2} \mid 2 \leq y_{1} \leq 10,3 \leq y_{2} \leq 24\right\}, F_{1}(x, y)=$ $h_{1}(x, y)=e^{T}(x-y), \forall x, y \in C, F_{2}(x, y)=h_{2}(x, y)=5 e^{T}(x-$ $y), \forall x, y \in Q$, and $A=\left(\begin{array}{ll}2 & 0 \\ 0 & 6\end{array}\right), B=\left(\begin{array}{cc}1 / 2 & 0 \\ 0 & 1 / 2\end{array}\right)$, where $e=(1,1)^{T}$.

It is easy to see that the optimal solution of Example 1 is $x^{*}=(5,4)^{T}$. Now, we use Algorithm 13 to compute the solution of the problem. Let $\alpha=1 / 2, \xi=1 / 7, D=\left(\begin{array}{cc}9 / 10 & 0 \\ 0 & 9 / 10\end{array}\right)$, $\gamma=1.2, r_{n}=1 / 10+1 / 2 n, \lambda_{n}=1 / n, \alpha_{n}=1 / \sqrt{n}, \beta_{n}=$ $1 / 10+1 / n^{2}, \gamma_{n}=1 / n^{2}$, and $f(x)=x / 2, \forall x \in H_{1}$. The stopping criterion is $\left\|x_{n+1}-x_{n}\right\| \leq \epsilon$. For $\epsilon=10^{-7}$ and different initial points which are presented randomly, such as

$$
\begin{aligned}
& x=\operatorname{rand}(2,1), \\
& x=10 * \operatorname{rand}(2,1), \\
& x=1000 * \text { rand }(2,1),
\end{aligned}
$$

separately. Table 1 shows the initial value, the final value, and the cpu time for the above three cases. We denote Init., Fina., and Sec. the initial value, the final value, and the cpu time in seconds, respectively.

From Table 1, we can see that the final value $\bar{x}$ is not influenced by the initial value.

To show the changing tendency of the final value $\bar{x}$ for different $\epsilon$, Table 2 gives the different $\epsilon$, the initial value, the final value, and the cpu time in seconds.

Now, we further express the status that the final value $\bar{x}$ tends to optimal solution $x^{*}$ through Figures 1 and 2 . We carry out 100 experiments for different $\epsilon$ from $10^{-7}$ to $10^{-9}$. From Figures 1 and 2, we know that the final value $\bar{x}$ tends to the optimal solution $x^{*}$ when $\epsilon$ tends to 0 , which illustrates the efficiency of Algorithm 13.

\section{Conclusion}

In this paper, we study the split generalized equilibrium problem and variational inequality problem. For finding their common solution, we propose a kind of conjugate gradient viscosity approximation algorithm. Under mild conditions, we prove that the sequence generated by the 
TABLE 2: The final value and the cpu time for different $\epsilon$.

\begin{tabular}{llll}
\hline$\epsilon$ & Init. & Fina. & Sec. \\
\hline $10^{-5}$ & $x=(8.0028,1.4189)^{T}$ & $\bar{x}=(4.9640,3.9712)^{T}$ & 0.08 \\
$10^{-6}$ & $x=(7.4313,3.9223)^{T}$ & $\bar{x}=(4.9835,3.9868)^{T}$ & 0.30 \\
$10^{-7}$ & $x=(5.4722,1.3862)^{T}$ & $\bar{x}=(4.9924,3.9939)^{T}$ & 1.35 \\
\hline
\end{tabular}

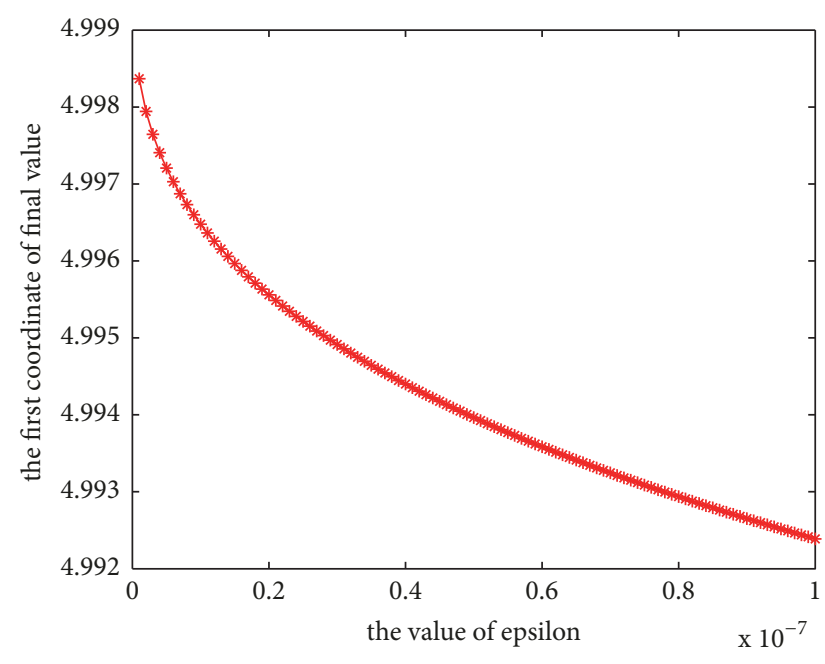

FIgURE 1: The behaviors of first coordinate of $\bar{x}$ for different $\epsilon$.

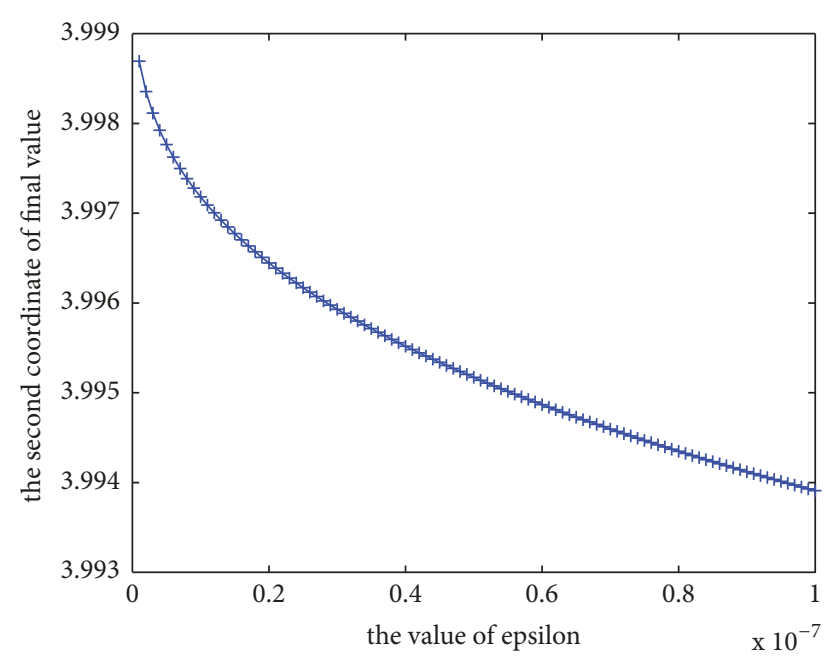

FIGURE 2: The behaviors of the second coordinate of $\bar{x}$ for different $\epsilon$.

iterative algorithm converges strongly to the common solution. In comparison to [25], the authors introduce and study an effective iterative algorithm to approximate a common solution of a split generalized equilibrium problem and a fixed point problem. Under suitable conditions, they proved a strong convergence theorem for the sequence generated by the iterative scheme. Furthermore, we can study the iterative algorithm for finding a common solution of the split generalized equilibrium problem, variational inequality problem, and fixed point problem.

\section{Data Availability}

The data used to support the findings of this study are included within the article.

\section{Conflicts of Interest}

The authors declare that there are no conflicts of interest regarding the publication of this paper.

\section{Acknowledgments}

This project is supported by the Natural Science Foundation of China (Grants nos. 11401438 and 11571120) and Shandong Provincial Natural Science Foundation (Grants nos. ZR2013FL032 and ZR2017LA002) and the Project of Shandong Province Higher Educational Science and Technology Program (Grant no. J14LI52).

\section{References}

[1] Y. Censor and T. Elfving, "A multiprojection algorithm using Bregman projections in a product space," Numerical Algorithms, vol. 8, no. 2-4, pp. 221-239, 1994.

[2] H. Che and M. Li, "The conjugate gradient method for split variational inclusion and constrained convex minimization problems," Applied Mathematics and Computation, vol. 290, pp. 426-438, 2016.

[3] S. Y. Cho, W. Li, and S. M. Kang, "Convergence analysis of an iterative algorithm for monotone operators," Journal of Inequalities and Applications, 2013:199, 14 pages, 2013.

[4] P. L. Combettes, "Solving monotone inclusions via compositions of nonexpansive averaged operators," Optimization. A Journal of Mathematical Programming and Operations Research, vol. 53, no. 5-6, pp. 475-504, 2004.

[5] F. Facchinei and J.-S. Pang, Finite-dimensional variational inequalities and complementarity problems, vol. 1, Springer, New York, NY, USA, 2003.

[6] N. Fang and Y. Gong, "Viscosity iterative methods for split variational inclusion problems and fixed point problems of a nonexpansive mapping," Communications in Optimization Theory, vol. 2016, 15 pages, 2016.

[7] Meixia Li, Xiping Kao, and Haitao Che, "A simultaneous iteration algorithm for solving extended split equality fixed point problem," Mathematical Problems in Engineering, vol. 2017, Article ID 9737062, 9 pages, 2017.

[8] B. Liu, B. Qu, and N. Zheng, "A successive projection algorithm for solving the multiple-sets split feasibility problem," Numerical Functional Analysis and Optimization, vol. 35, no. 11, pp. 1459-1466, 2014.

[9] H. Mahdioui and O. Chadli, "On a System of Generalized Mixed Equilibrium Problems Involving Variational-Like Inequalities in Banach Spaces: Existence and Algorithmic Aspects," 
Advances in Operations Research, vol. 2012, Article ID 843486, 18 pages, 2012.

[10] X. Qin, B. A. Bin Dehaish, and S. Y. Cho, "Viscosity splitting methods for variational inclusion and fixed point problems in Hilbert spaces," Journal of Nonlinear Sciences and Applications. JNSA, vol. 9, no. 5, pp. 2789-2797, 2016.

[11] B. Qu and H. Chang, "Remark on the successive projection algorithm for the multiple-sets split feasibility problem," Numerical Functional Analysis and Optimization, vol. 38, no. 12, pp. 1614-1623, 2017.

[12] B. Qu, B. Liu, and N. Zheng, "On the computation of the stepsize for the CQ-like algorithms for the split feasibility problem," Applied Mathematics and Computation, vol. 262, pp. 218-223, 2015.

[13] X. Wang, "Alternating proximal penalization algorithm for the modified multiple-sets split feasibility problems," Journal of Inequalities and Applications, vol. 48, pp. 1-8, 2018.

[14] M. Zhang, "Strong convergence of a viscosity iterative algorithm in Hilbert spaces," Journal of Nonlinear Functional Analysis, vol. 2014, 16 pages, 2014.

[15] H. Zhang and Y. Wang, "A new CQ method for solving split feasibility problem," Frontiers of Mathematics in China, vol. 5, no. 1 , pp. 37-46, 2010.

[16] W. Takahashi and M. Toyoda, "Weak convergence theorems for nonexpansive mappings and monotone mappings," Journal of Optimization Theory and Applications, vol. 118, no. 2, pp. 417428, 2003.

[17] A. S. Antipin, "Methods for solving variational inequalities with related constraints," Computational Mathematics and Mathematical Physics, vol. 40, no. 9, pp. 1291-1307, 2000.

[18] H. Che, Y. Wang, and M. Li, "A smoothing inexact Newton method for $P_{0}$ nonlinear complementarity problem," Frontiers of Mathematics in China, vol. 7, no. 6, pp. 1043-1058, 2012.

[19] H. Chen and Y. Wang, "A family of higher-order convergent iterative methods for computing the Moore-Penrose inverse," Applied Mathematics and Computation, vol. 218, no. 8, pp. 40124016, 2011.

[20] G. M. Korpelevich, "An extragradient method for finding saddle points and for other problems," Ekonomika i Matematicheskie Metody, vol. 12, no. 4, pp. 747-756, 1976.

[21] S. Lian and L. Zhang, "A simple smooth exact penalty function for smooth optimization problem," Journal of Systems Science \& Complexity, vol. 25, no. 3, pp. 521-528, 2012.

[22] M. Sun, Y. Wang, and J. Liu, "Generalized Peaceman-Rachford splitting method for multiple-block separable convex programming with applications to robust PCA," Calcolo. A Quarterly on Numerical Analysis and Theory of Computation, vol. 54, no. 1, pp. 77-94, 2017.

[23] G. Wang and H.-t. Che, "Generalized strict feasibility and solvability for generalized vector equilibrium problem with setvalued map in reflexive Banach spaces," Journal of Inequalities and Applications, vol. 66, pp. 1-11, 2012.

[24] B. Zhong, H. Dong, L. Kong, and J. Tao, "Generalized nonlinear complementarity problems with order $P_{0}$ and $R_{0}$ properties," Positivity. An International Mathematics Journal Devoted to Theory and Applications of Positivity, vol. 18, no. 2, pp. 413-423, 2014.

[25] K. R. Kazmi and S. H. Rizvi, "Iterative approximation of a common solution of a split generalized equilibrium problem and a fixed point problem for nonexpansive semigroup," Mathematical Sciences, vol. 7, pp. 1-10, 2013.
[26] S. Chang, "Some problems and results in the study of nonlinear analysis," Nonlinear Analysis, vol. 30, no. 7, pp. 4197-4208, 1997.

[27] K. Shimoji and W. Takahashi, "Strong convergence to common fixed points of infinite nonexpansive mappings and applications," Taiwanese Journal of Mathematics, vol. 5, no. 2, pp. 387404, 2001.

[28] T. Suzuki, "Strong convergence theorems for infinite families of nonexpansive mappings in general Banach spaces," Fixed Point Theory and Applications, vol. 2005, no. 1, pp. 103-123, 2005.

[29] G. Marino and H. K. Xu, "A general iterative method for nonexpansive mappings in Hilbert spaces," Journal of Mathematical Analysis and Applications, vol. 318, no. 1, pp. 43-52, 2006.

[30] L. S. Liu, "Ishikawa and Mann iterative process with errors for nonlinear strongly accretive mappings in Banach spaces," Journal of Mathematical Analysis and Applications, vol. 194, no. 1, pp. 114-125, 1995.

[31] H. K. Xu, "Viscosity approximation methods for nonexpansive mappings," Journal of Mathematical Analysis and Applications, vol. 298, no. 1, pp. 279-291, 2004.

[32] C. Byrne, "A unified treatment of some iterative algorithms in signal processing and image reconstruction," Inverse Problems, vol. 20, no. 1, pp. 103-120, 2004.

[33] C. Martinez-Yanes and H. Xu, "Strong convergence of the CQ method for fixed point iteration processes," Nonlinear Analysis: Theory, Methods \& Applications, vol. 64, no. 11, pp. 2400-2411, 2006.

[34] C. Byrne, Y. Censor, A. Gibali, and S. Reich, “The split common null point problem," Journal of Nonlinear and Convex Analysis, vol. 13, no. 4, pp. 759-775, 2012.

[35] A. Moudafi, "The split common fixed-point problem for demicontractive mappings," Inverse Problems, vol. 26, 6 pages, 2010.

[36] R. T. Rockafellar, "Monotone operators and the proximal point algorithm," SIAM Journal on Control and Optimization, vol. 14, no. 5, pp. 877-898, 1976. 


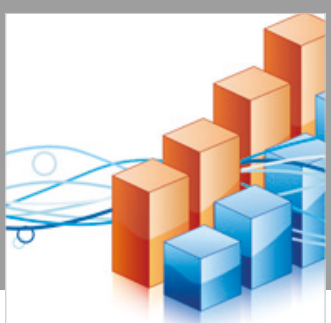

Advances in

Operations Research

\section{-n-m}
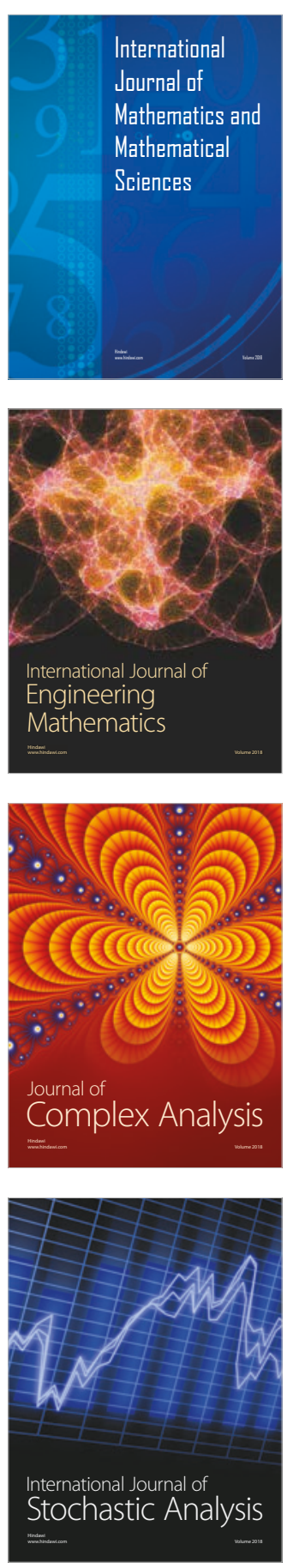
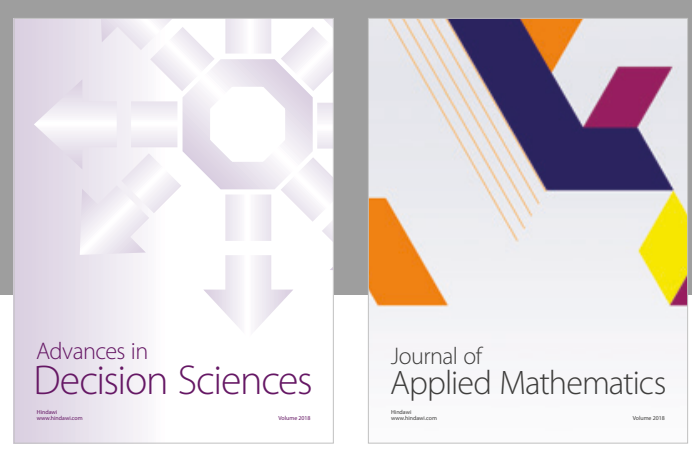

Journal of

Applied Mathematics
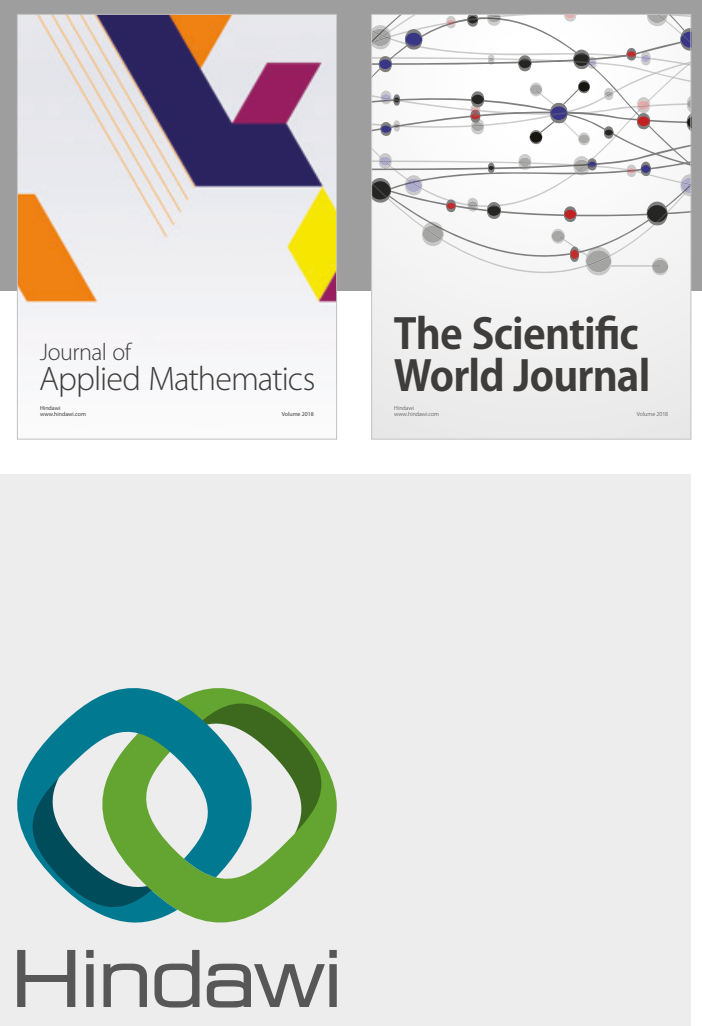

Submit your manuscripts at

www.hindawi.com

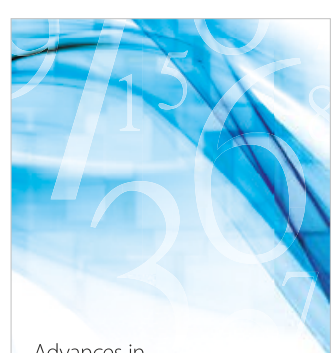

Advances in
Numerical Analysis
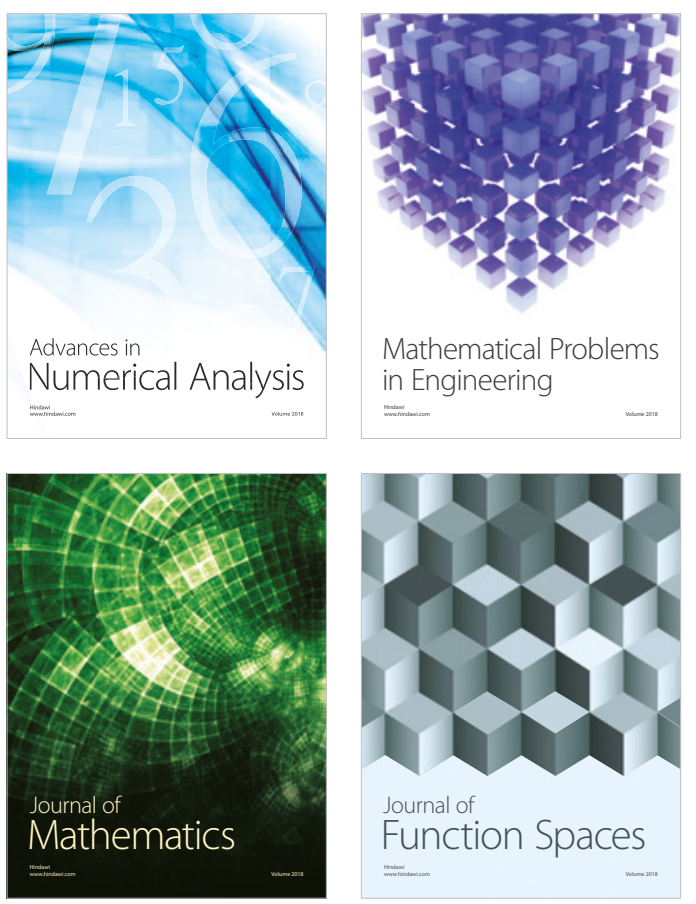

Mathematical Problems in Engineering

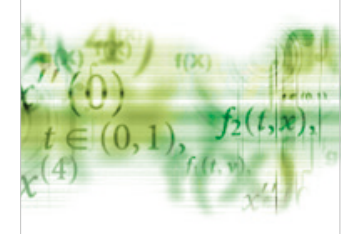

International Journal of

Differential Equations

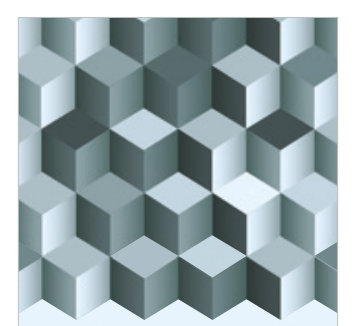

Journal of

Function Spaces

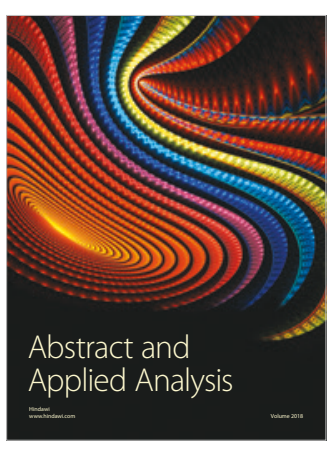

The Scientific

World Journal

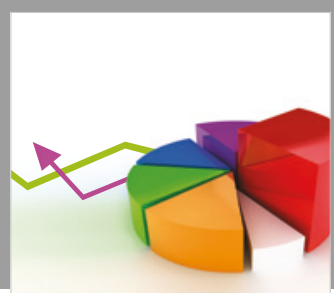

Journal of

Probability and Statistics
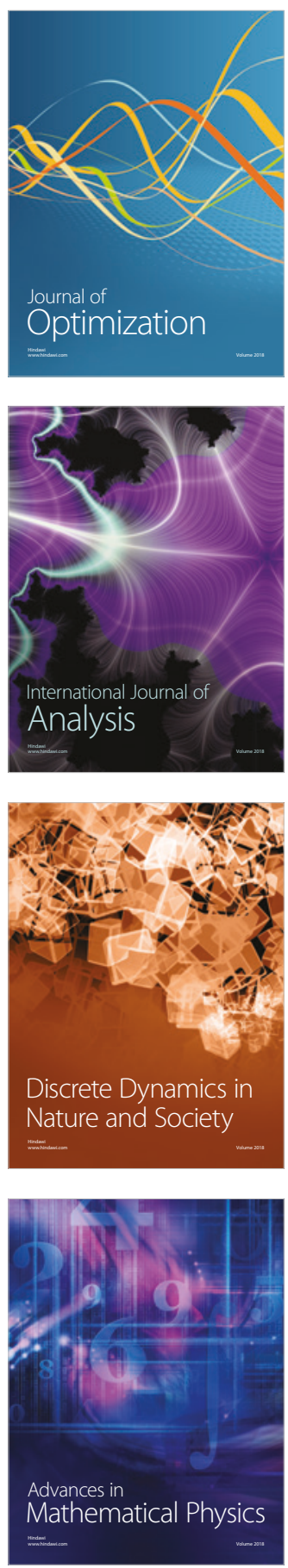